COMMUNICATIONS IN

ANALYSIS AND GEOMETRY

Volume 4, Number 2, 285-331, 1996

\title{
Surfaces convexes dans des espaces lorentziens à courbure constante
}

\author{
JEAN-MARC SchlENKER
}

\begin{abstract}
Résumé
Nous étudions les immersions isométriques de surfaces à courbure $K<$ $K_{0}$ dans des espaces lorentziens de dimension 3 à courbure constante, et en particulier les dégénérescences de suites de telles immersions. Nous en déduisons des résultats d'existence et d'unicité d'immersions isométriques de surfaces compactes dans l'espace de Sitter, ainsi que des résultats d'existence quand les surfaces sont seulement complètes.
\end{abstract}

\begin{abstract}
We study isometric immersions of surfaces with curvature $K<K_{0}$ in 3 -dimensionnal Lorentz spaces with constant curvature $K_{0}$, and more specifically degeneracies of sequences of such immersions. Then we use those results to prove existence and unicity for some isometric immersions of compact surfaces in the de Sitter space, as well as existence results for complete surfaces.
\end{abstract}

\section{Introduction et résultats.}

Soit $(\Sigma, \sigma)$ une surface munie d'une métrique à courbure $K<K_{0}$ et $(M, \mu)$ un espace lorentzien de dimension 3 à courbure constante égale à $K_{0}$. Nous allons étudier les immersions isométriques de $(\Sigma, \sigma)$ dans $(M, \mu)$.

Ce problème d'immersion isométrique est dit "uniformément elliptique", car l'opérateur différentiel qui décrit l'immersion l'est. La courbure extrinsèque de l'immersion (qui peut se calculer à partir des courbures sectionnelles de $\Sigma$ et $M$ par la formule de Gauss) est uniformément positive, et la surface immergée est localement convexe. Voir [Gro86] pour de nombreux 
résultats concernant les immersions isométriques dans des espaces riemanniens et pseudo-riemanniens.

Le cas où la variété $M$ est riemannienne et non lorentzienne a été étudié en détail, d'abord par Pogorelov (cf. [Pog73]) et Nirenberg (cf. [Nir53]). Plus tard, M. Gromov (cf. [Gro85]) a mis a point une méthode basée sur les "courbes pseudo-holomorphes" particulièrement efficace pour résoudre certains problèmes elliptiques sur des surfaces. On pourra trouver dans [Pan] un exposé très lisible de certains aspects de cette méthode. L'utilisation des courbes pseudo-holomorphes a permis à F. Labourie (cf. [Lab89] [Lab87] et surtout [Lab94]) de simplifier considérablement et de généraliser les résultats antérieurs concernant les immersions isométriques convexes dans les espaces $M$ riemanniens. Dans le cas où $M$ est l'espace de Minkowski de dimension 3 , les immersions isométriques de surfaces compactes de type espace sont impossibles, mais d'intéressants résultats existent quand même, voir part exemple [SG85], [Sok79].

Nous allons commencer par donner quelques exemples typiques de dégénérescences de suites d'immersions uniformément elliptiques dans des espaces lorentziens à courbure constante. Puis nous verrons comment, malgré l'absence de compacité du problème, on peut encore appliquer des méthodes de courbes pseudo-holomorphes quand l'espace $M$ est à courbure constante ; nous nous concentrerons sur le cas où $M$ est l'espace lorentzien simplement connexe à courbure constante $1, S_{1}^{3}$. Cet espace peut s'obtenir comme une "pseudo-sphère" dans l'espace de Minkowski de dimension 4, soit $\left\{x \in R_{1}^{4} \mid\langle x \mid x\rangle=1\right\}$, muni de la métrique induite par celle de $R_{1}^{4}$. Cf. [O'N83] pour la géométrie lorentzienne, et [CM62] pour les principales propriété de $S_{1}^{3}$, etc...

En particulier, il existe une "dualité" entre $S_{1}^{3}$ et $H^{3}$, qui permet d'associer à un 2-plan de $S_{1}^{3}$ un point de $H^{3}$ et à un 2-plan de $H^{3}$ un point de $S_{1}^{3}$ (en passant par l'opération qui à un 2-plan de $R_{1}^{4}$ associe son 2-plan orthogonal). Ainsi, on associe à une immersion elliptique d'une surface dans $H^{3}$ une immersion elliptique de la même surface dans $S_{1}^{3}$, et réciproquement. De plus, la première forme fondamentale (c'est à dire la métrique induite) de chacune de ces immersions est la troisième forme fondamentale de l'autre. Cette dualité ne sera pas utilisée ici (sauf pour le lemme 5.6, où on peut facilement s'en passer) mais est importante pour comprendre le problème; consulter [RH93] pour plus de détails sur ce sujet.

Nous allons trouver des similitudes avec le cas riemannien, mais aussi des différences importantes. En particulier, des dégénérescences de suites d'immersions uniformément elliptiques de surfaces compactes peuvent se produire. Il existe encore un résultat décrivant les limites sans 
dégénérescences de suites d'immersions dans un espace lorentzien simplement connexe à courbure constante $M$ :

Théorème 5.5. Soit $\phi_{n}: D \rightarrow M$ une suite d'immersions de type espace uniformément elliptiques du disque telles que $\left(\phi^{*} g_{0}\right)_{n \in N}$ converge $C^{\infty}$ vers une métrique $g_{\infty}$ et que la suite des courbures moyennes $H_{n}$ soit (uniformément) d'intégrale majorée, et soit $\left(x_{n}\right)_{n \in N}$ telle que $\left(x_{n}\right)$ et $\left(j^{1} \phi_{n}\left(x_{n}\right)\right)$ convergent. Alors il existe une suite extraite de $\phi_{n}$ qui converge $C^{\infty}$ vers une immersion isométrique.

Mais il faut aussi un résultat décrivant les dégénérescences; c'est le:

Théorème 5.6. Soit $\left(f_{n}\right)_{n \in N}: D \rightarrow M$ une suite d'immersions de type espace uniformément elliptiques du disque, telle que $\left(f_{n}^{*} g_{0}\right)_{n \in N}$ converge $C^{\infty}$ vers $g_{\infty}$. Soit $\left(x_{n}\right)_{n \in N}$ une suite convergeant vers $x_{\infty}$ telle que $\left(j^{1} f_{n}\left(x_{n}\right)\right)$ converge, mais sans que $\left(f_{n}\right)$ converge $C^{\infty}$ au voisinage de $x_{\infty}$. Alors il existe une suite extraite de $\left(f_{n}\right)_{n \in N}$, qu'on notera encore $\left(f_{n}\right)_{n \in N}$, une géodésique maximale $\gamma \ni x_{\infty}$ de $\left(D, g_{\infty}\right)$ et un segment géodésique $\Gamma$ de $M$, tels que $\left(f_{n \mid \gamma}\right)$ converge vers une isométrie sur $\Gamma$.

Ce phénomène "empêche" certaines surfaces d'admettre une immersion isométrique dans $S_{1}^{3}$. On va démontrer en particulier un énoncé proposé par C. D. Hodgson et I. Rivin dans [RH93] (voir aussi [Riv86]) concernant les immersions de sphères:

Théorème 7.3. Soit $\sigma$ une métrique sur $S^{2}, x_{0} \in S^{2}, y_{0} \in S_{1}^{3}$, et soit $I \in \operatorname{Isom}\left(T_{x_{o}} S^{2}, T_{y_{0}} S_{1}^{3}\right)$; alors $\left(S^{2}, \sigma\right)$ admet un plongement isométrique $f$ uniformément elliptique dans $S_{1}^{3}$, telle que que $j^{1} f\left(x_{0}\right)=I$, si et seulement si

- $\left(S^{2}, \sigma\right)$ n'admet pas de géodésique fermée de longueur $L \leq 2 \pi$;

- la courbure de $\left(S^{2}, \sigma\right)$ vérifie partout $K<1$.

Il existe alors exactement deux tels plongements distincts.

Ce résultat est lié au théorème d'Andreev et aux résultats de Hodgson et Rivin sur les polyèdres convexes de $H^{3}$ (cf. [Riv86, RH93]) dont il peut se voir comme une version lisse. En effet, la dualité qui existe entre $H^{3}$ et $S_{1}^{3}$ montre que l'étude des immersions isométriques elliptiques de surfaces dans $S_{1}^{3}$ correspond à l'étude des immersions de surfaces convexes dans $H^{3}$ à troisième forme fondamentale donnée ; quand on remplace les surfaces par des polyèdres, on constate que les polyèdres convexes de $S_{1}^{3}$ à métrique 
(longueur des cotés) donnée correspondent aux polyèdres convexes à angles dièdraux donnés dans $H^{3}$, d'où la relation au théorème d'Andreev [And70] ; on pourra consulter [Riv86] ou [RH93] pour ces questions. Ainsi, le théorème 7.3 peut se formuler en disant qu'une métrique $\tilde{\sigma}$ sur la sphère $S^{2}$ est la troisième forme fondamentale d'une immersion dans $H^{3}$ si et seulement si sa courbure est strictement majorée par 1 et si elle n'admet pas de géodésique fermée de longueur inférieure à $2 \pi$.

Au passage, la démonstration de ce théorème nous mènera à des résultats un peu en marge des autres (cf. la section 7 pour les définitions). Le premier est le :

Théorème 7.4. Soit $\phi: V^{k} \rightarrow S_{1}^{n}$ une immersion elliptique de type espace d'une variété complète dans $S_{1}^{n}, n \geq k \geq 1$. Alors la courbure sectionnelle de $\left(V, \phi^{*} g_{0}\right)$ est partout inférieure strictement à 1 (quand $k \geq 2$ ), et on a: $\operatorname{Vol}_{k}(V) \geq \operatorname{Vol}_{k}\left(S^{k}, c a n\right)$.

mais on peut aussi donner un résultat de non-existence d'immersions isométriques en codimension 1 sous des hypothèses de courbure de Ricci :

Théorème 7.5. Soit $\left(V^{n}, \nu\right)$ une variété riemannienne (connexe) de dimension $n$ (éventuellement à bord, ouverte,etc...) à courbure de Ricci vérifiant :

$$
\forall v \in V, \forall x \in T_{v} V, \operatorname{Ric}(x, x)<(n-1) \nu(x, x)
$$

Supposons que $(V, \nu)$ admet une géodésique fermée de longueur $L \leq 2 \pi$. Alors $(V, \nu)$ n'admet pas d'immersion isométrique dans $S_{1}^{n+1}$.

L'interêt principal de ces deux résultat est qu'on peut espérer qu'ils se généralisent au cas de certaines espaces lorentziens à courbure variable.

Ensuite, l'utilisation des résultats concernant les surfaces compactes nous permettra de passer au cas de surfaces complètes et d'obtenir le:

Théorème 8.5. Soit $(\Omega, \omega)$ une surface riemannienne complète telle que:

1. $\Omega$ admet un plongement régulier dans $S^{2}$;

2. $K(\omega) \leq 1-\epsilon$ pour un certain $\epsilon>0$;

3. $(\Omega, \omega)$ n'admet pas de géodésique fermée de longueur $L \leq 2 \pi+\epsilon$;

4. la borne inférieure des longueurs des courbes fermées non homotopes $\grave{a} 0$ de $(\Omega, \omega)$ est strictement supérieure à $2 \pi$. 
Surfaces convexes dans des espaces lorentziens à courbure constante 289

Alors $(\Omega, \omega)$ admet au moins deux immersions isométriques elliptiques dans $S_{1}^{3}$ ayant un 1-jet donné en un point donné.

Enfin nous finirons par une sorte de réciproque à ce résultat, qui montre que les hypothèses faites ne sont pas déplacées:

Théorème 8.6. Soit $(S, \sigma)$ une surface complète, dans laquelle il existe une courbe fermée non homotopiquement triviale de longueur inférieure strictement à $2 \pi$. Alors $(S, \sigma)$ n'admet pas d'immersion isométrique elliptique dans $S_{1}^{3}$.

Il est clair que la seule surface compacte admettant une immersion de type espace dans $S_{1}^{3}$ est $S^{2}$; en effet, $S_{1}^{3}$ peut s'écrire sous la forme $S^{2} \times R$ avec la métrique $\operatorname{ch}(r)^{2} \operatorname{can}_{S^{2}}-d r^{2}, r$ étant la coordonnée sur $R$, et donc toute surface $S$ immergée de type espace est un graphe (de pente majorée par $\operatorname{ch}(r)$ ) au-dessus de $S^{2}$; si $S$ est compacte et connexe, $S$ est donc difféomorphe à $S^{2}$. Nous nous limiterons donc, chaque fois qu'on aura affaire à une surface compacte de type espace immergée dans $S_{1}^{3}$, à considérer le cas de $S^{2}$. Le même raisonnement nous montre d'ailleurs qu'une surface compacte connexe de type espace immergée dans $S_{1}^{3}$ est toujours plongée.

Il faut enfin remarquer que les démonstrations d'existences d'immersions isométriques présentées ici sont essentiellement $C^{\infty}$ à cause de l'utilisation de résultats sur les courbes pseudo-holomorphes, qui sont énoncés dans ce cadre. Par contre, les énoncés de non-existence sont valable dans un cadre moins régulier. Il n'est pas évident (contrairement au cas où l'espace où se fait l'immersion est riemannien) d'en déduire des résultats dans des catégories moins régulières par approximation. Par contre, les résultats polyèdraux de [RH93] laissent supposer que des résultat moins régulier sont peut-être vrais.

Les résultats présentés ont été obtenus sous la direction de François Labourie; il a une grande part dans les idées nouvelles qu'on pourra trouver ici. Je tiens aussi a remercier I. Rivin pour les intéressantes remarques qu'il m'a communiquées. 


\section{Exemples de dégénérescences}

Dans le cas où l'espace où se font les immersions est riemannien et non lorentzien, les dégénérescences de suites d'immersions isométriques uniformément elliptiques sont bien comprises (cf. [Lab87]). Deux phénomènes sont remarquables : d'une part, même quand une suite d'immersions isométriques (convergeant en un point) ne converge pas $C^{\infty}$, elle admet une sous-suite qui converge $C^{0}$. En effet, la métrique riemannienne assure que l'image d'un compact par l'immersion reste dans un compact.

D'autre part, François Labourie [Lab87] a montré qu'il ne pouvait pas exister de dégénérescence de suites d'immersions uniformément elliptique d'une surface compacte.

Nous allons montrer par l'étude de deux exemples simples que la situation correspondant ä un espace "cible" lorentzien est plus complexe.

Commençons par étudier une suite d'immersions du disque dans $\mathbf{R}_{1}^{3}$. On peut partir d'une immersion "symétrique" du disque comme ci-dessous :

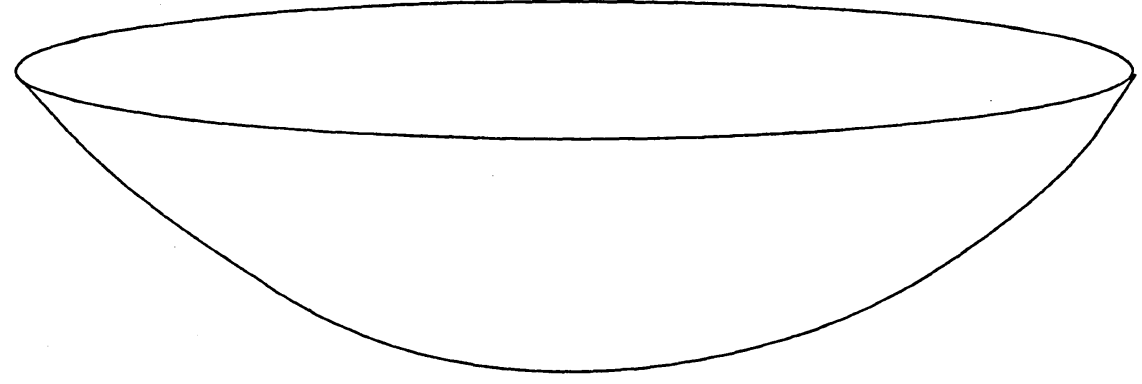

Figure 1.a

Augmentons maintenant l'une des courbures principales tout en diminuant l'autre (en gardant le produit constant pour satisfaire à la formule de Gauss). La surface immergée se déforme de la façon suivante :

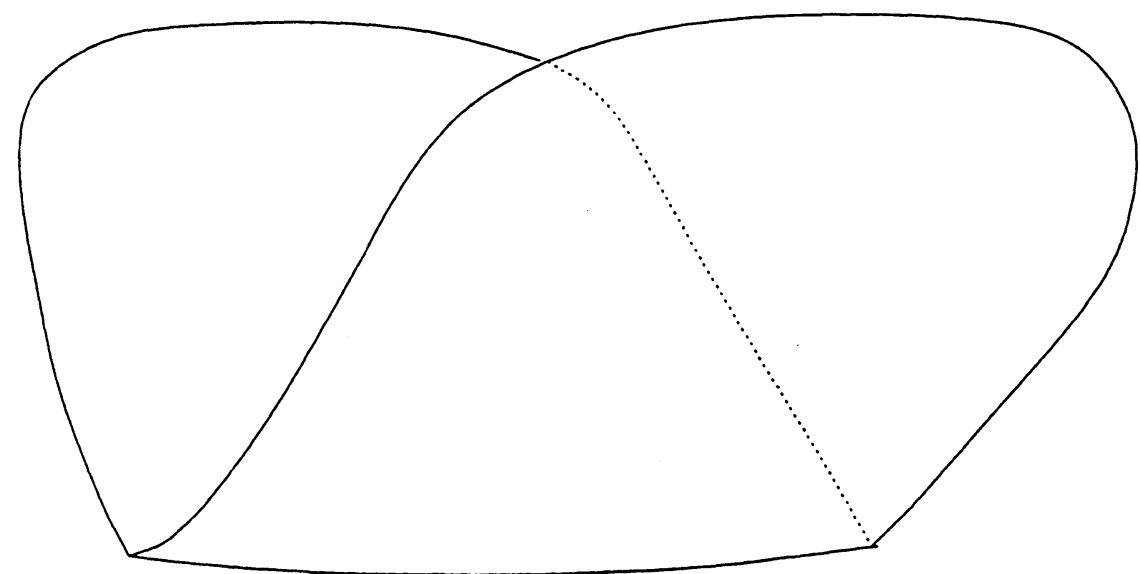

Figure 1.b 
Si on mène ce processus jusqu'à son terme, on voit apparaitre à la limite une surface qui est l'intersection suivant une géodésique de type espace de deux surfaces totalement géodésiques tangentes au cône de lumière :

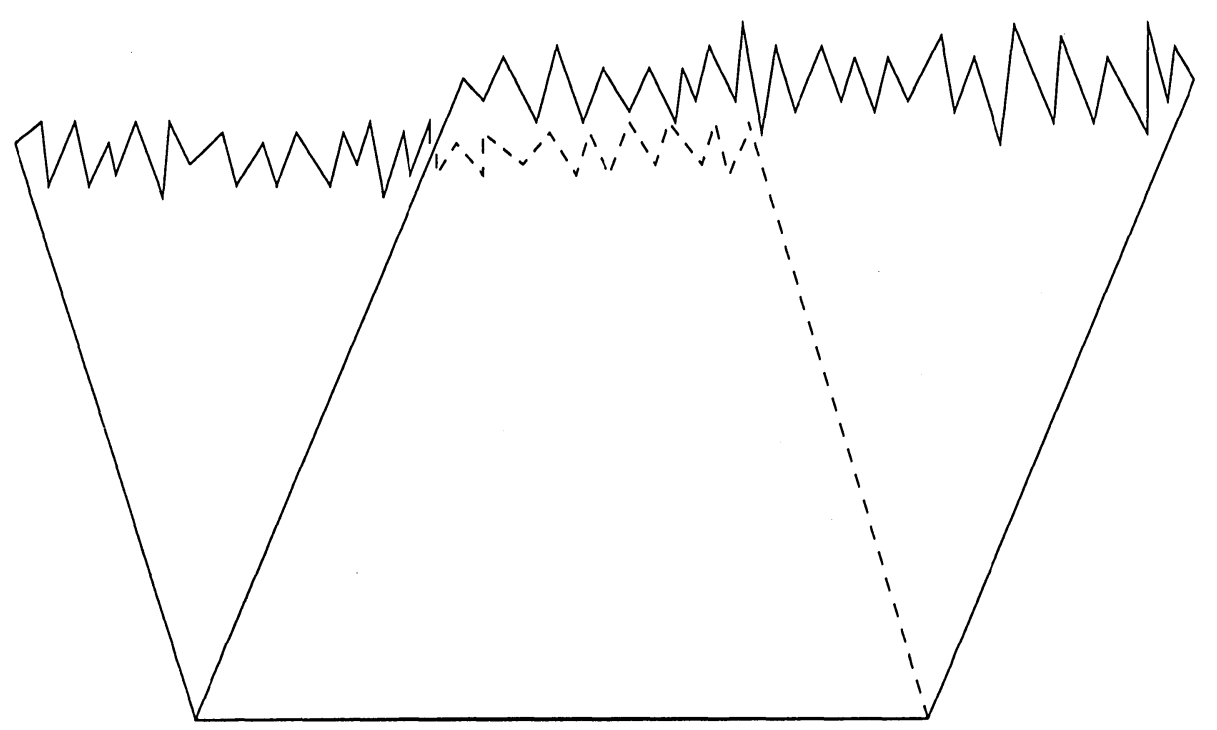

Figure 1.c

On constate dans cet exemple qu'on a pas de convergence $C^{0}$ : en effet, à l'exception des images réciproques de la géodésique de pli, tous les points du disque sont envoyés par la limite à l'infini. Ceci correspond à la proximité des plans tangents à l'image des immersions et du cône de lumière de $R_{1}^{3}$.

Par ailleurs, on peut avoir dégénérescence de suites d'immersions uniformément elliptiques de surfaces compactes. Ce phenomène apparait dans $S_{1}^{3}$ (qui est essentiellement le seul espace lorentzien à courbure constante dans lequel on peut faire des immersions elliptiques de surfaces compactes) mais nous allons l'aborder par l'intermédiaire de $H^{3}$, en utilisant la dualité entre $H^{3}$ et $S_{1}^{3}$ qu'on a rappelée dans l'introduction.

Considérons donc la suite de surfaces obtenues de la façon suivante : 


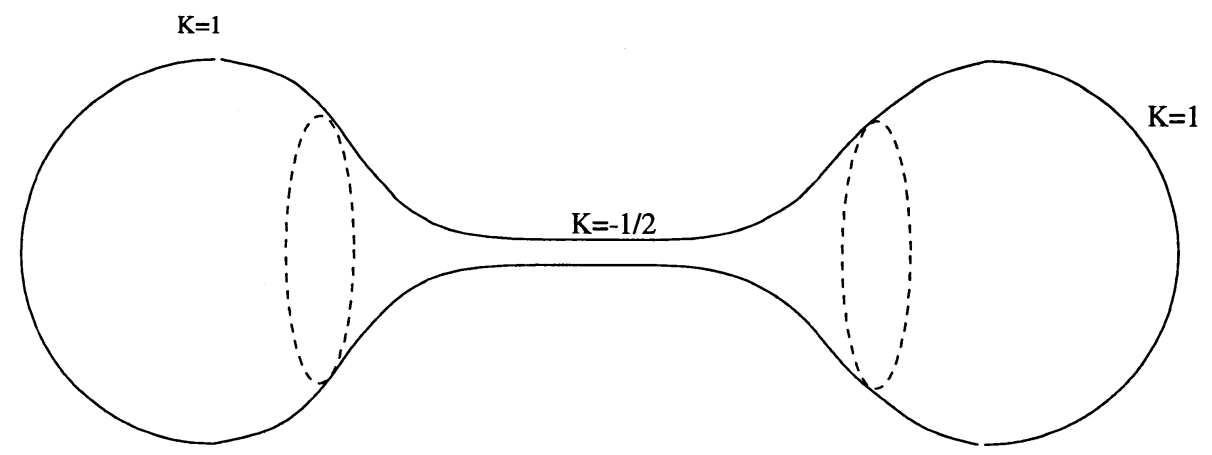

Figure 2.a

On a joint deux morceaux de sphère (de courbure 1) par un "cylindre" hyperbolique de courbure $-1 / 2$ dont on peut faire tendre la longueur vers l'infini. Comme ces surfaces sont à courbure $K>-1$, elles admettent des immersions isométriques elliptiques dans $H^{3}$, dont on peut exiger par exemple qu'elles soient symétriques par rapport à un point fixé de $H^{3}$.

Un point intéressant est qu'on vérifie facilement (en utilisant les symétries de ce modèles ainsi que les bornes d'aire) que les troisièmes formes fondamentales de ces immersions convergent vers une métrique du type suivant :

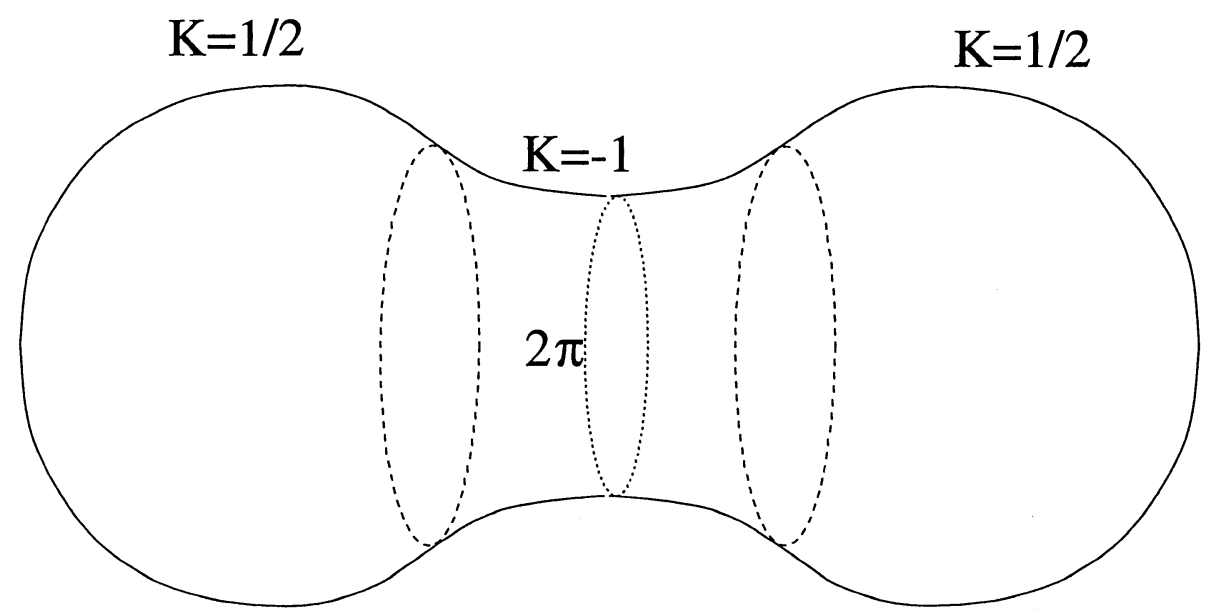

Figure 2.b

Par contre, comme les deux "sphères" de la surface de la figure 2.a tendent vers l'infini dans $H^{3}$, les images des immersions par la dualité de $H^{3}$ dans $S_{1}^{3}$ dégénérent dans $S_{1}^{3}$. On peut vérifier que, dans cet exemple, la dégénérescence se produit le long de l' "équateur" de longueur $2 \pi$ dans la 
figure $2 . \mathrm{b}$; nous montrerons dans la suite de ce chapitre que ce phénomène est général, et que toutes les dégénérescences ressemblent à celle ci.

\section{Courbes pseudo-holomorphes.}

Les outils qu'on va présenter ici ont été développés par M. Gromov (cf. [Gro85]) pour étudier les problèmes hyperboliques sur les surfaces. Ils sont valables dans un cadre beaucoup plus général que celui où nous allons utiliser, et nous ne ferons appel qu'à certaines de leurs intéressantes propriétés. Mais nous allons rappeler de façon assez complète, dans cette section, la démonstration des énoncés que nous utiliserons car nous sommes dans un cadre légèrement différent de celui où ils sont d'habitude présentés, en particulier en ce qui concerne les arguments de compacité : nous allons utiliser des objets équivariants dans des espaces non compacts.

Commençons par rappeler quelques définitions classiques:

Définition 3.1. Une structure presque-complexe sur une variété $V$ est un automorphisme de fibrés vectoriels $J: T V \rightarrow T V$ tel que $J^{2}=-I . J$ sera dite calibrée par une 1-forme $\beta$ si

$$
\forall x \in T V, d \beta(x, J x)>0
$$

Si $V$ est munie d'une structure riemannienne dont la restriction à $P$ est $J$-hermitienne, on dira que $J$ est $\epsilon$-calibrée $(\epsilon>0)$ par $\beta$ si

$$
\forall x \in T V, d \beta(x, J x) \geq \epsilon\|x\|^{2}
$$

et si de plus

$$
\forall x \in T V, \beta(x) \leq \frac{1}{\epsilon}\|x\|
$$

Si $P$ est une une distribution de $p$-plans, on dira que $\beta$ calibre $J$ respectivement à $P$ si $J(P) \subset P$ et si les identités ci-dessus sont vraies lorsque $x \in P$.

Les variétés presque complexes sont donc des généralisations immédiates des variété complexes: on n'exige pas de condition d'intégrabilité de $J$. La calibration peut être vue comme une généralisation de la 1-forme de Liouville d'une forme de Kähler.

Introduisons les courbes pseudo-holomorphes de M. Gromov (cf. [Gro85]): 
Définition 3.2. Soit $(V, J)$ une variété munie d'une structure presque complexe et $\left(S, J^{\prime}\right)$ une surface de Riemann, c'est à dire une surface munie d'une structure complexe. Une application $\phi$ de $S$ dans $V$ est pseudo-holomorphe si

$$
\forall v \in T S, \phi_{*}\left(J^{\prime} v\right)=J\left(\phi_{*}(v)\right)
$$

Elles correspondent aux courbes holomorphes quand la variété d'arrivée est complexe et non pas seulement presque complexe. Le miracle qui les rend utiles est que beaucoup des propriété des courbes holomorphes, et en particulier leur régularité, sont préservées; la reconnaissance de ce fait fondamental est due à $\mathrm{M}$. Gromov, qui l'a utilisé en particulier pour montrer un résultat de dégénérescence de courbes pseudo-holomorphes (cf. [Gro85]).

En associant à un problème elliptique une structure presque complexe sur un espace bien choisi, de manière à ce que les solutions correspondent à des courbes pseudo-holomorphes, on peut au moins dans certains cas obtenir des résultats par des méthodes géométriques; voir [Gro85] pour d'autres exemples que celui présenté ici.

Nous donnons ici une version "équivariante" du "lemme de Schwarz" de Gromov; l'introduction d'une action de groupe va nous permettre d'utiliser le "gros" groupe d'isométries de $S_{1}^{3}$ pour compenser son absence de compacité.

Lemme de Schwarz. Soit $G$ un groupe de Lie et $E \rightarrow F$ un $G$-fibré principal sur une variété compacte, muni:

- d'une distribution de p-plans $V \subset T E$

- d'une structure presque-complexe $J$ définie sur $V$ (donc à valeurs dans $V)$

- d'une métrique $\mu$ dont la restriction à $V$ est $J$-hermitienne

ces trois objets étant invariants par l'action de G. Soit $\phi$ une fonction pseudo-holomorphe de $D$, le disque unité de $C$, dans une région $\epsilon$-calibrée de $E$ ( $\phi$ est donc telle que pour $x \in D, \phi_{*}\left(T_{x} D\right) \subset V$ ). Alors les dérivées de $\phi$ en 0 sont majorées a priori.

Pour démontrer ce lemme, nous allons suivre la méthode de P. Pansu dans [Pan], et en particulier utiliser trois propositions qui sont proches de certaines de celles qui se trouvent dans l'article cité. La première permet de se ramener à la recherche d'inégalités isopérimétriques (elle provient intégralement et presque mot pour mot de [Pan]): 
Surfaces convexes dans des espaces lorentziens à courbure constante 295

Proposition 3.3. Soit $(S, \sigma)$ une surface riemannienne, $r>0$, et $I: R_{+} \rightarrow$ $R_{+}$son "profil isopérimétrique", c'est à dire que

$$
\forall r \in R_{+}, I(r)=\sup \left\{s \in R_{+} \mid \forall \Omega \subset S,(\operatorname{Aire}(\Omega) \geq r) \Rightarrow(L(\partial \Omega) \geq s)\right\}
$$

Supposons que I soit tel que:

1. au voisinage de $0, I$ est au moins euclidienne, plus précisément

$$
I(a)^{2} \leq \frac{4 \pi a}{1+\epsilon(a)}
$$

avec

$$
\int_{0} \epsilon(a) \frac{d a}{a}<\infty
$$

2. au voisinage de l'infini, I est strictement mieux que euclidienne, plus précisément

$$
\int^{\infty} I(a)^{-2} d a<\infty
$$

Alors pour toute immersion conforme $f: D \rightarrow S$ du disque unité dans $S$, on $a$

$$
\left|f^{\prime}(0)\right| \leq C(I)
$$

où $C$ est une constante ne dépendant que de I.

Démonstration. (Cf. [Gro85] ou [Pan]) Notons $a(r)=\operatorname{Aire}\left(f\left(D_{r}\right)\right)$, où $D_{r}=$ $\{z \in C|| z \mid \leq r\}$, et $\psi$ une primitive de $I^{-2}$. On a alors

$$
a^{\prime}(r)=\int_{\partial D_{r}}\left|f^{\prime}\right|^{2} \geq \frac{1}{2 \pi r}\left(\int_{\partial D_{r}}\left|f^{\prime}\right|\right)^{2} \geq \frac{I(a(r))^{2}}{2 \pi r}
$$

et donc par intégration, si $s<t$,

$$
4 \pi \psi(a(t))-\log \left(\pi t^{2}\right) \geq 4 \pi \psi(a(s))-\log \left(\pi s^{2}\right)
$$

Mais d'après l'hypothèse (1),

$$
\int_{1}^{a} I(u)^{-2} d u \geq \int_{1}^{a} \frac{d u}{4 \pi u}+\int_{1}^{a} \epsilon(u) \frac{d u}{4 \pi u}
$$

et puisque

$$
\int_{0} \epsilon(a) \frac{d a}{a}<\infty
$$


on voit qu'on peut ajouter une constante à $\psi$ pour avoir

$$
\lim _{a \rightarrow 0} 4 \pi \psi(a)-\log (a)=0
$$

On a alors

$$
\log \left|f^{\prime}(0)\right|=\lim _{s \rightarrow 0} \log \frac{a(s)}{\pi s^{2}}=\lim _{t \rightarrow 0}\left[4 \pi \psi(a(t))-\log \left(\pi t^{2}\right)\right]
$$

donc

$$
\log \left|f^{\prime}(0)\right| \leq \lim _{t \rightarrow 1}\left[4 \pi \psi(a(t))-\log \left(\pi t^{2}\right)\right] \leq 4 \pi \psi(\infty)
$$

d'où le résultat d'après l'hypothèse (2).

Il nous suffit donc, pour montrer le lemme 2.2.3, de trouver deux inégalités isopérimétriques. D'abord, pour $a$ petit, on utilise une majoration de la courbure des surfaces pseudo-holomorphes dans $E$ (cf. encore [Gro85] ou $[\mathrm{Pan}])$ :

Proposition 3.4. Dans la situation du lemme 2.2.3, la courbure moyenne d'une surface pseudo-holomorphe $S$ dans $E$ est majorée par une constante. La courbure (intrinsèque) de $S$ pour $\mu_{\mid S}$ est aussi donc majorée par une constante.

Démonstration. (provenant encore de [Pan]) On peut sans difficulté compléter $J$ et $\mu$ en une structure presque complexe et en une métrique $J$ hermitienne toutes deux $G$-invariante sur $E$ tout entier. On a alors la formule suivante pour la courbure moyenne d'une surface pseudo-holomorphe dans $E$ :

$$
H=\operatorname{tr}(\bar{\partial} J)_{\mid S}
$$

et comme $H$ ne dépend donc que des dérivées (premières) de $J$, on a par compacité de $E / G$ et par invariance de $J$ par l'action de $G$ que $H$ est uniformément majorée. Mais on en déduit alors grâce à la formule de Gauss et à la majoration de la courbure sectionnelle $K$ de $E$ (qui provient encore de la compacité de $E / G$ et de l'invariance de $\mu$ par l'action de $G$ ) que la courbure $\kappa$ de $S$ vérifie:

$$
\kappa \leq K+\frac{1}{2}|H|^{2} \leq \text { Const }
$$

Utilisons maintenant l'hypothèse de calibration pour trouver une autre inégalité isopérimétrique, utile cette fois quand $a \rightarrow \infty$ : 
Proposition 3.5. Dans la situation du lemme 2.2.3, soit $S$ une courbe pseudo-holomorphe dans $E, \Omega$ un domaine de $S$ inclus dans une région $\epsilon$-calibrée de $E$ et $\partial \Omega$ son bord. On a alors:

$$
\operatorname{Aire}(\Omega) \leq \epsilon^{-2} L(\partial \Omega)
$$

Démonstration. Comme $\beta_{\mid S} \epsilon$-calibre une région de $S$ contenant $\Omega$, on a:

$$
\operatorname{Aire}(\Omega) \leq \epsilon^{-1} \int_{\Omega} d \beta=\epsilon^{-1} \int_{\partial \Omega} \beta \leq \epsilon^{-2} \mathrm{~L}(\partial \Omega)
$$

Démonstration. (du lemme 2.2.3) On a trouvé les deux inégalités isopérimétriques nécessaires pour obtenir, d'après la proposition 2.4 , une majoration de la dérivée première de $\phi$ vue comme application conforme de $D$ dans $\left(S, \mu_{\mid T S}\right)$, c'est à dire comme application pseudo-holomorphe de $\left(D, J_{0}\right)\left(J_{0}\right.$ étant la structure presque complexe naturelle de $D)$ dans $(S, J)$. Il reste à remarquer qu'il existe une structure presque complexe naturelle sur l'espace des 1-jets d'immersions de $D$ dans $E$, qui rend pseudo-holomorphes les 1jets des immersions pseudo-holomorphes; on obtient donc une majoration de la dérivée seconde de $\phi$ en 0 , etc ... Le lecteur curieux pourra pour cela consulter [Gro85] ou [Pan].

Il nous reste à rappeler deux lemmes techniques provenant de [Lab89], qui précisent le comportement à la limite de suites d'immersions pseudoholomorphes dans une variété presque complexe.

Notons pour cela $E$ une variété, $G_{2}(E)$ la grassmannienne de ses 2-plans; si $f: D \rightarrow E$ est une immersion, notons $\bar{f}: D \rightarrow G_{2}(E)$ l'application naturelle déduite. Supposons $E$ munie d'une suite $J_{n}$ de structures presque complexe convergeant $C^{\infty}$ vers $J_{\infty}$, et d'une suite $\mu_{n}$ de métriques $J_{n^{-}}$ hermitiennes convergeant $C^{\infty}$ vers $\mu_{\infty}$. On a alors le résultat de nondégénérescence suivant, extrait sans modification de [Lab89], qui contient en plus sa démonstration:

Proposition 3.6. Soit $\left(f_{n}\right)_{n \in N}$ une suite d'immersions $J_{n}$-pseudo-holomorphes de $D$ dans $\left(E, J_{n}\right)$, convergeant $C^{\infty}$ sur les compacts vers $f_{\infty}$ non constante, telle que $\left(\bar{f}_{n}\right)_{n \in N}$ converge. Alors $f_{\infty}$ est une immersion. 
En termes simples, ceci signifie que le fait que les $f_{n}$ soient pseudoholomorphes suffit pour assurer que, si ils admettent une limite en tant qu'applications à valeurs dans la grassmannienne des 2-plans, alors cette limite est soit complètement dégénérée (la limite des $f_{n}$ étant constante) soit au contraire partout régulière (la limite des $f_{n}$ est une immersion). Continuons avec un lemme de [Lab89] concernant le comportement à la limite d'une suite d'immersions pseudo-holomorphes du disque :

Proposition 3.7. Si l'aire des $f_{n}(D)$ est uniformément bornée, alors $f_{\infty}(\partial D)$ est inclus dans la limite de Hausdorff des $f_{n}(\partial D)$.

La démonstration de cette proposition qui se trouve dans [Lab89] reste valable ici, bien qu'on se trouve dans un cas un peu différent.

Enfin voici un dernier lemme tiré mot pour mot de [Lab89] (qui le présente comme un résultat de [Gro85]) :

Proposition 3.8. Soit $\pi: F \rightarrow E$ un fibré muni d'une connexion sur une variété presque-complexe $E$, et dont la fibre est kählerienne. On munit l'espace total de la structure presque-complexe induite. Soit $\left(h_{n}\right)$ une suite d'applications pseudo-holomorphe de $D$ dans $F$ telles que:

1. $\left(\pi \circ h_{n}\right)$ converge;

2. les $h_{n}(D)$ sont inclus dans un compact $K$;

3. $K$ possède un voisinage $U$ dont l'intersection avec chaque fibre est de $H^{2}$ de nul.

Alors les dérivées des $h_{n}$ sont majorées à priori.

Cet énoncé nous permettra d'éviter les problèmes dus à l'apparition possible de singularités sur les limites de suites de courbes pseudo-holomorphes convergentes. Sa démonstration est d'ailleurs une conséquence assez facile de ce qui précède : la calibration correspond à la 1-forme de Kähler (avec la condition topologique) et la compacité permet d'utiliser le lemme de Schwarz, la version équivariante n'étant d'ailleurs pas nécessaire.

\section{Structure presque complexe.}

Nous allons voir comment utiliser les outils de la section précédente pour étudier les immersions elliptiques de surfaces dans un espace lorentzien $M$ de dimension 3 simplement connexe à courbure sectionnelle constante (cette 
hypothèse n'est pas absolument indispensable, mais c'est ce cas qui nous sera utile dans la suite). L'essentiel de cette section est similaire à ce qu'on peut trouver dans [Lab89].

Nous allons commencer par introduire des notations concernant les espaces qui nous seront utiles par la suite:

Définition 4.1. On choisit une surface riemannienne compacte (avec ou sans bord) $(S, \sigma)$ et on notera $E$ le fibré $\operatorname{Isom}(T S, T M)$ au-dessus de $S \times M$.

On remarquera que le groupe $\operatorname{Isom}(M)$ agit de manière naturelle sur $E$, et que comme cette action est transitive sur les 2-plans de $T M$ de type espace, $E / \operatorname{Isom}(M)$ est homéomorphe à $S$. On est donc dans la situation du lemme de Schwarz sous la forme énoncée plus haut.

Précisons maintenant la structure de $E$ en suivant [Lab89] ou [Lab94]:

Proposition 4.2. En tout point $g: T_{s} S \rightarrow T_{m} M$ de $E$, on peut écrire de manière canonique: $T_{g} E \simeq T_{s} S \times T_{m} M \times T_{m} M$.

Démonstration. (cf. [Lab89]) Il suffit de remarquer que si on se donne $g=$ $(s, m, u) \in S \times M \times \operatorname{Isom}\left(T_{s} S, T_{m} M\right)$, alors on peut construire simplement un difféomorphisme d'un voisinage de $(s, m, e) \in S \times M \times S O(2,1)$ sur un voisinage de $g$ dans $E$, en utilisant l'action naturelle de $S O(2,1)$ sur $T_{m^{\prime}} M \simeq R_{1}^{3}$ et en envoyant $\left(s^{\prime}, m^{\prime}, \alpha\right) \in S \times M \times S O(2,1)$ sur

$$
\begin{gathered}
g^{\prime}: T_{s^{\prime}} S \rightarrow T_{m^{\prime}} M \\
x \mapsto\left(\Pi_{m}^{m^{\prime}} \circ \alpha \circ u \circ \Pi_{s^{\prime}}^{s}\right)(x)
\end{gathered}
$$

où $\Pi_{m}^{m^{\prime}}$ désigne le transport parallèle le long des géodésiques de longueur minimimale de $m$ à $m^{\prime}$ dans $M$ et de $s$ à $s^{\prime}$ dans $S$. Puis on vérifie qu'en passant aux espaces tangents, on a bien l'identification annoncée car $T_{m} M \simeq T_{e} S O(2,1)$ (l' identification peut se faire en passant par les "produits vectoriels" car la métrique de $T_{m} M$ est non dégénérée).

Nous allons continuer à suivre [Lab89] et définir dans le cas où l'immersion est elliptique (ce qui revient à imposer $K_{S}<1$ partout) une distribution de 4-plans qui jouera un rôle fondamental:

Définition 4.3. (cf. [Lab89]) En tout point $g: T_{s} S \rightarrow T_{m} M$ de $E$, on note $k=\sqrt{K_{0}-K_{S}(s)}$, où $K_{0}$ est la courbure sectionnelle de $M$, et on pose

$$
V=\left\{H(u, v)=(u, g(u), k g(v)) \mid u, v \in T_{s} S\right\} \subset T_{g} E
$$


On définit de plus une structure presque-complexe sur $V$ par

$$
J H(u, v)=H(v,-u)
$$

et une métrique $\mu$ sur $V$ par

$$
\mu\left(H(u, v), H\left(u^{\prime}, v^{\prime}\right)\right)=k\left(\sigma\left(u, u^{\prime}\right)+\sigma\left(v, v^{\prime}\right)\right)
$$

On remarque alors que si $f: S \rightarrow M$ est une immersion, on peut en déduire $\tilde{f}=j^{1} f: S \rightarrow E ; \operatorname{Im}(T \tilde{f})$ est alors partout tangent à $V$, et $\tilde{f}(S)$ est $J$-pseudo-holomorphe (en tant que courbe non paramétrée). On peut d'ailleurs vérifier facilement que la structure complexe $\tilde{f}^{*} J$ induite par $\tilde{f}$ sur $S$ coincide avec celle qui est définie par la troisième forme fondamentale III de l'immersion. En fait, la condition de tangence à $V$ et celle de $J$-pseudoholomorphie correspondent, ensemble, aux conditions d'holonomie qui expriment qu'une application de $S$ dans $E=\operatorname{Isom}(T S, T M)$ est le 1-jet d'une immersion elliptique de $S$ dans $M$; le fait que ces conditions d'holonomie prennent une forme pseudo-holomorphe est lié au caractère elliptique du problème. Mais on va voir qu'il existe d'autre surfaces pseudo-holomorphes possibles dans $E$, qui apparaîtront comme limites "dégénérées" de 1-jets de suites d'immersions elliptiques.

L'introduction des 4-plans $V$ correspond au fait que tout l'espace n'est pas "utile": en fait, les applications de $S$ dans $E$ qu'on va considérer sont induites par des immersions isométriques de $S$ dans $M$, ce qui implique des conditions d'holonomie qui correspondent entre autres à la tangence à ces 4plans; mais comme ils ne forment pas une distribution intégrable, on ne peut pas vraiment se ramener à une variété plus petite. Par ailleurs, la version du lemme de Schwarz qui se trouve plus haut nous autorise à ne définir les quantité importantes que sur $V$, c'est à dire là où elles ont une signification géométrique.

On notera dans la suite $M_{K_{0}}$ l'espace simplement connexe à courbure sectionnelle constante égale à $K_{0}$, et pour $x \in M_{K_{0}}, N_{x}$ sera l'ensemble des points $y \in M_{K_{0}}$ tels que les géodésiques de longueur minimale joignant $x$ à $y$ soient de type espace et de longueur au plus $\pi / 2 \sqrt{K_{0}}$ (on vérifie facilement que, quel que soit $K_{0} \in R$, toutes les géodésiques reliant $x$ et $y$ sont de même type, et que si $y \in N_{x}$ il existe alors une unique dont la longueur est minimale).

Définissons maintenant un automorphisme de fibré $J^{\prime}$ sur $p_{M}^{*} T M$ ("tiré" par la projection $p_{M}: E \rightarrow M$ de $T M$ vu comme fibré sur $M$ ) en posant que la restriction de $J^{\prime}$ à $p_{s}^{*}(T S)$ (qu'on peut voir comme un sous fibré de $p_{M}^{*} T M$ grâce à l'immersion) est la structure complexe $J_{\sigma}$ associée à $\sigma$, et que 
Surfaces convexes dans des espaces lorentziens à courbure constante 301

la restriction de $J^{\prime}$ au fibré normal à $p_{s}^{*}(T S)$ dans $p_{M}^{*} T M$ pour la métrique induite par $\mu$ sur $p_{M}^{*} T M$ est l'identité. On peut alors poser (presque) comme dans [Lab89]:

Définition 4.4. On définit un champ de vecteurs $h_{x}$ sur $N_{x}$ par

$$
h_{x}(m)=\exp _{m}^{-1}(x)
$$

et une 1-forme $\beta_{x}$ sur $E$ au-dessus de $N_{x}$ par

$$
\beta_{x}(u, v, w)=-\operatorname{det}\left(h_{x}, n, J^{\prime} w\right)
$$

pour $(u, v, w) \in T_{g} E, n$ étant le vecteur normal unitaire $(\langle n \mid n\rangle=-1)$ à $\operatorname{Im}(g) \subset T M$.

La calibration se fait comme dans le cas riemannien; nous aurons pour cela besoin d'une hypothèse d'ellipticité uniforme des immersions: la courbure $K$ de $S$ doit vérifier $K \leq 1-\epsilon^{\prime}$ pour un $\epsilon^{\prime}>0$ fixé (ce qui revient à imposer $k \geq k_{0}>0$ ). La proposition suivante ainsi que sa démonstration sont alors proches d'un lemme du à F. Labourie dans [Lab89]:

Proposition 4.5. $J$ est $\epsilon$-calibrée respectivement à $V$ dans la région de $E$ au-dessus de $N_{x}$ par $d \beta_{x}$ pour un certain $\epsilon>0$.

Démonstration. (cf. [Lab89]) Par hypothèse, $k \geq k_{0}>0$, ce qui correspond à l'ellipticité uniforme. D'abord si $(u, v, w) \in T_{s} S \times T_{m} M \times T_{m} M$ alors

$$
\left|\beta_{x}(u, v, w)\right|=\left|\operatorname{det}\left(h_{x}, n, J^{\prime} w\right)\right| \leq\left\|h_{x}\right\| \cdot\|w\|
$$

donc

$$
\left|\beta_{x}(u, v, w)\right| \leq \pi\|w\| \leq \frac{\pi}{k_{0}} \sqrt{\mu((u, v, w),(u, v, w))}
$$

Ensuite, si $\left(u_{1}, v_{1}, w_{1}\right),\left(u_{2}, v_{2}, w_{2}\right) \in T_{s} S \times T_{m} M \times T_{m} M$ avec $m \in N_{x}$, alors

$$
d \beta_{x}\left(\left(u_{1}, v_{1}, w_{1}\right),\left(u_{2}, v_{2}, w_{2}\right)\right)=-\operatorname{det}\left(v_{1}, n, J^{\prime} w_{2}\right)+\operatorname{det}\left(v_{2}, n, J^{\prime} w_{1}\right)
$$

et donc si $m \in N_{x}, g: T_{s} S \rightarrow T_{m} M$ et $\nu \in V \subset T_{g} E$, on peut écrire $\nu$ sous la forme $\nu=H(u, v)=(u, g u, k g v)$ avec $u, v \in T_{s} S$;on a $J \nu=H(v,-u)=$ $(v, g v,-k g u)$ et donc :

$$
\begin{aligned}
d \beta_{x}(\nu, J \nu) & =-\operatorname{det}\left(u, n, k J^{\prime}(-u)\right)+\operatorname{det}\left(v, n, k J^{\prime} v\right) \\
& =k\left(\|u\|_{\sigma}^{2}+\|v\|_{\sigma}^{2}\right) \\
& =\mu(\nu, \nu)
\end{aligned}
$$


et donc $J$ est bien $\epsilon$-calibrée par $d \beta_{x}$ pour un $\epsilon>0$ lié à $k_{0}$.

On a même en fait un peu plus: $\beta_{x}$ est invariante par l'action de $G$. Mais ce fait ne nous sera pas utile dans la suite. Par contre, $\beta_{x}$ ne calibre $J$ que respectivement à $V$ mais c'est suffisant. L'intérêt de cette proposition réside dans ce que l'image par une immersion isométrique uniformément elliptique d'un disque de rayon assez petit est toujours contenu dans $N_{x}$, où $x$ est l'image du centre du disque.

Enfin le résultat technique suivant est nécessaire pour utiliser le lemme de Schwarz dans les cas qui nous intéresseront:

Proposition 4.6. Soit $(S, \sigma)$ avec $K(\sigma) \leq 1-\epsilon, x \in S, y \in E$; alors il existe $A, B>0$ tels que pour toute immersion isométrique $f: S \rightarrow M$ telle que $j^{1} f(x)=y$, il existe un voisinage $U$ de $x$ dans $S$, homéomorphe au disque, tel que:

- $\int_{U} H \leq A$;

- $d_{E}\left(j^{1} f(x), j^{1} f(\partial U)\right) \geq B$.

la distance dans $E$ étant définie comme la borne inférieure des longueurs des chemins allant d'un point $\grave{a}$ un autre en étant partout tangents aux 4-plans $V$ où la métrique est définie.

Démonstration. Par étapes:

- Notons $\Sigma_{\alpha}$ l'ensemble des points de $M$ qui peuvent être joints à $f(x)$ par un segment géodésique (de type temps) de longueur $-\alpha$. On peut définir dans un voisinage compact $K^{\prime} \subset E$ de $j^{1} f(x)$ une application $n: K^{\prime} \rightarrow \Sigma_{\alpha}$ (pour $\alpha$ assez petit) en envoyant un $y \in K^{\prime}$ sur la première intersection avec $\Sigma_{\alpha}$, dans la direction de la normale orientée à $S$, de la géodésique partant de $\pi_{M}(y)$, (la projection de $y$ sur $M$ ) avec pour vitesse $N(y)$, la normale unitaire à $\operatorname{Im}(y)$. 
Surfaces convexes dans des espaces lorentziens à courbure constante 303

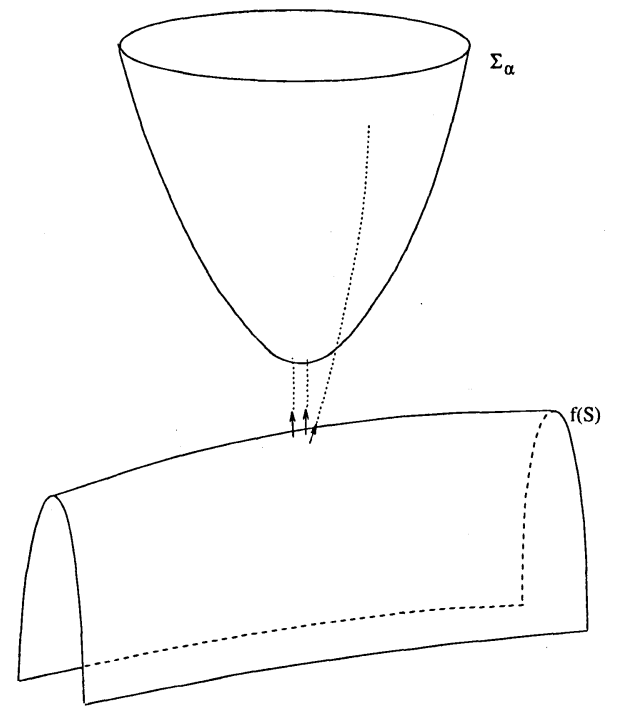

Figure 3

- Si on choisit $K^{\prime}$ assez petit, il existe une constante $C_{1}>0$ telle que pour tout $\xi \in T E$ de la forme $\xi=H(u, v)$, l'angle orienté (sur $S$ ) entre $u$ et $v$ étant dans $[0, \pi]$, on ait:

$$
\left\|n_{*} \xi\right\| \geq C_{1} \cdot\|\xi\|
$$

Il suffit pour le voir de constater que cette propriété est vraie dans le cas où l'espace d'arrivée est $R_{1}^{3}$, et est stable par petites déformations de la métrique.

- Notons $S^{\prime}=\pi_{S}\left(K^{\prime}\right)$. Il existe alors une constante $C_{2}>0$ telle que

$$
\forall z \in T S^{\prime},\left\|\left(n \circ j^{1} f\right)_{*} z\right\| \geq C_{2} \cdot\|z\|
$$

En effet, pour $z \in T S$, on a: $\left(j^{1} f\right)_{*}(z)=H(z, J z)$ qui est bien de la forme $H(u, v)$ avec un angle orienté entre $u$ et $v$ dans $[0, \pi]$, et on déduit (2) de (1) et de la forme de la métrique $\mu$.

- Considérons la fonction

$$
\begin{gathered}
K^{\prime 2} \backslash\left\{(y, y) \mid y \in K^{\prime}\right\} \rightarrow R \\
(y, z) \mapsto d_{E}(y, z) / d_{\Sigma_{\alpha}}(n(y), n(z))
\end{gathered}
$$

On vérifie que cette application est continue, et minorée au voisinage de $\left\{(y, y) \mid y \in K^{\prime}\right\}$; elle admet donc une borne inférieure $C_{3}>0$ par compacité de $K^{\prime}$. 
- Pour $\alpha, \beta$ assez petits, l'image de $n \circ j^{1} f$ contient $B_{\beta}\left(\left(n \circ j^{1} f\right)(x)\right) \subset \Sigma_{\alpha}$ grâce à (2); on peut donc poser $U=\left(n \circ j^{1} f\right)^{-1}\left(B_{\beta}\left(\left(n \circ j^{1} f\right)(x)\right)\right)$ et on a par définition de $C_{3}$ que

$$
d_{E}(x, \partial U) \geq C_{3} \cdot \beta
$$

- Enfin on vérifie que $\int_{U} H \leq A$ car le jacobien de l'identité vue comme application de $(S, I)$ dans $\left(S,\left(j^{1} f\right)^{*} \mu\right)$ est $H . k$, et l'aire pour $\mu$ de $\left(j^{1} f\right)(U)$ est majorée par $\operatorname{Aire}\left(B_{\beta}\left(\left(n \circ j^{1} f\right)(x)\right)\right) / C_{1}^{2}$ d'après (1).

\section{Dégénérescence d'immersions elliptiques.}

L'étude de ces dégénérescences permet de compléter l'espace des immersions isométriques d'une surface dans $M$; elle nous sera utile pour démontrer des résultats d'existence d'immersions isométriques .

Nous allons suivre ici en partie la démarche de [Lab89], à ceci près que des suites d'immersions de surfaces compactes peuvent dégénérer, ce qui n'était pas possible dans le cas riemannien. Les résultats présentés ici peuvent être rapprochés de ceux, plus généraux, de Labourie [Lab].

Nous allons commencer par introduire (comme dans [Lab89]) certaines surfaces pseudo-holomorphes de $E$ qui sont justement celles qui ne proviennent pas d'immersions elliptiques ; mais elles peuvent être limite de courbes déduites d'immersions : elles correspondent au "pli" d'une surface le long d'une géodésique de $M$.

Définition et proposition. Soit $\gamma$ une géodésique maximale de $(S, \sigma), \Gamma$ une géodésique maximale de $M$ de type espace, et $\rho: \tilde{\gamma} \rightarrow \Gamma$ une isométrie du revêtement universel $\tilde{\gamma}: I \rightarrow M$ de $\gamma$ sur $\Gamma$. La surface de $E$ définie par

$$
\Sigma_{(\gamma, \Gamma)}=\cup_{s \in I}\left\{g \in \operatorname{Isom}\left(T_{\tilde{\gamma}(s)} S, T_{\rho(\tilde{\gamma}(s))} M\right) \mid g_{*}\left(\gamma^{\prime}(s)\right)=\Gamma^{\prime}\right\}
$$

est pseudo-holomorphe; on l'appellera surface de pli associée à $\gamma, \Gamma$ et $\rho$.

Démonstration. On vérifie sans mal que si $\sigma \in \Sigma$ alors

$$
T_{\sigma} \Sigma=\left\{H\left(\lambda \gamma^{\prime}, \mu \gamma^{\prime}\right) \mid \lambda, \mu \in R\right\}
$$

donc $T_{\sigma} \Sigma \subset V$ et $T_{\sigma} \Sigma$ est invariant par $J$. 
Surfaces convexes dans des espaces lorentziens à courbure constante 305

On pourra trouver d'intéressantes considérations sur ces surfaces de pli, ainsi que sur d'autres aspects spécifiques des équations de Monge-Ampère, dans [Gou96] ; il y est en particulier montré que, dans un cadre assez général, ce sont des solutions particulières des équations qu'on peut "échanger" avec des solutions "usuelles" en faisant agir sur l'espace des 1-jets de fonctions sur une surface des transformations de contact (pour la structure canonique de cet espace de 1-jets) qui se trouvent laisser invariantes la classe des équations de Monge-Ampère telle que la définit [Gou96]. Cet aspect des équations de Monge-Ampère permet de mieux comprendre par exemple pourquoi on a des résultats de régularité pour les convergences vers des solutions qui semblent au premier abord très dégénérées...

Toujours comme dans [Lab89], nous allons montrer que les "surfaces de pli" correspondent aux seules limites non régulières possibles de suites d'immersions. Pour commencer, nous montrerons qu'il n'existe pas de point isolé où la courbure moyenne $H$ devient infinie.

Plaçons nous pour cela en un point où $H$ est assez grande pour imposer que les deux courbures principales soient distinctes, et notons $\pi_{S}$ la projection de $V$ sur $T\left(\left(j^{1} f\right) S\right), \omega$ la 1-forme de connexion du repère des directions principales (le repère orthonormé qui diagonalise $I$ ) et $W=1 / H$. On a la

Proposition 5.1. $W$ est solution sur $(S, I I)$ de:

$$
d W \circ J=W \eta+\left(1-4 k^{2} W^{2}\right) \pi_{S}^{*} \omega
$$

ainsi que de

$$
\left.d(d W \circ J)-d W \wedge \eta=W\left(d \eta-8 k \pi_{S}^{*} \omega \wedge d(k W)\right)+\left(1-4 K^{2} W^{2}\right) \pi_{S}^{*} \Omega\right)
$$

où $\eta$ est une 1-forme ne dépendant que de $\pi_{S}$.

Démonstration. Le lecteur est invité à se référer à [Lab89], propositions 3.6 et 3.7.

Nous allons maintenant nous placer dans le cas correspondant à l'étude d'une dégénérescence d'une suite d'immersions, c'est à dire que nous noterons:

- $S$ une surface;

- $\left(f_{n}\right)_{n \in N}$ une suite d'immersions $\epsilon$-elliptiques de $S$ dans $M$;

- $\left(g_{n}\right)_{n \in N}=\left(f_{n}^{*} g_{0}\right)_{n \in N}$ et $g_{\infty}$ sa $C^{\infty}$-limite, elle aussi supposée exister; 
- $\left(J_{n}\right)_{n \in N}$ la suite des structures complexes induites sur $S$ par les deuxièmes formes fondamentales des $f_{n}$;

- $\left(H_{n}\right)_{n \in N}$ la suite des courbure moyennes associées, et $W_{n}=1 / H_{n}$;

- $\left(x_{n}\right)_{n \in N}$ une suite de $S$ convergeant vers $x_{\infty}$ et telle que $\left(j^{1} f_{n}\left(x_{n}\right)\right)$ converge dans $E$.

On a (comme dans [Lab89]) la

Proposition 5.2. $\left(W_{n}\right)$ admet une sous suite qui converge $C^{\infty}$ vers une fonction $W_{\infty}$, qui est soit toujours, soit jamais nulle.

Démonstration. (cf. [Lab89]) On va utiliser certains des résultats rappelés plus haut. Choisissons un point $p_{0} \in S \backslash \partial S$. On va montrer qu'une suite d'applications du disque $D$ dans $E$, déduite de $\left(j^{1} f_{n}(S)\right)_{n \in N}$, admet une sous-suite qui converge $C^{\infty}$ dans un voisinage de $j^{1} f_{n}\left(p_{0}\right)$ vers une immersion pseudo-holomorphe. On en déduira que $\left(W_{n}\right)_{n \in N}$ converge $C^{\infty}$ en l'interprétant comme le déterminant de la projection de $j^{1} f_{n}(S)$ sur $S$.

Choisissons encore un point $y_{0} \in \operatorname{Isom}\left(T_{p_{0}} S, T M\right)$ et notons $\gamma_{n}$ une suite d'éléments de $G$ tels que $\gamma_{n} j^{1} f_{n}\left(p_{0}\right)=y_{0}$; c'est possible car $G=\operatorname{Isom}(M)$ agit transitivement sur le fibré des repères orthonormés de $M$. On commence par utiliser le lemme 4.6 pour déduire des $f_{n}$ des voisinages $O_{n}$ de $p_{0}$ tels que pour des $A, B$ indépendants de $n$, on ait

$$
\int_{O_{n}} H_{n} \leq A
$$

et

$$
d_{E}\left(j^{1} f_{n}\left(p_{0}\right), j^{1} f_{n}\left(\partial O_{n}\right)\right) \geq B
$$

On peut donc introduire des applications pseudo-holomorphes $\tilde{f}_{n}$ de $D$ dans $E$ données par la représentation conforme du disque $D$ dans $O_{n}$ suivie de l'application $j^{1} f_{n}$ et enfin de l'action de $\gamma_{n}$. De plus, on peut utiliser la calibration fournie par la proposition 4.5 et le lemme de Schwarz 2.2.3 montre que les dérivées des $\tilde{f}_{n}$ sont majorées (indépendemment de $n$ ) en tous les points intérieurs de $D$.

Or on a choisi la suite $\left(\gamma_{n}\right)_{n \in N}$ de façon à ce que $\gamma_{n} \cdot j^{1} f_{n}\left(p_{0}\right)$ converge, et la majoration sur les dérivées des $\tilde{f}_{n}$ montre que l'image des compacts de $D$ contenant $p_{0}$ par les $\tilde{f}_{n}$ reste dans une boule de $E$ (encore pour la 
Surfaces convexes dans des espaces lorentziens à courbure constante 307

distance définie par la borne inférieure des longueurs des chemins joignant deux points et tangents à $V$ ).

On peut donc appliquer à la suite $\left(\tilde{f}_{n}\right)_{n \in N}$ le théorème d'Ascoli sur les compacts de $D$, et en extraire une sous-suite $C^{\infty}$-convergente (vers une limite $\tilde{f}_{\infty}$ ) à laquelle nous nous restreindrons dorénavant.

Comme $H_{n}$ est le rapport des éléments d'aires entre $(S, \sigma)$ et $j^{1} f_{n}(S)$ munie de la métrique induite par $\mu,(5.1)$ montre que l'aire des $\tilde{f}_{n}(D)$ est uniformément bornée. On peut donc appliquer la proposition 3.7, et $\tilde{f}_{\infty}(\partial D)$ est contenue dans la limite de Hausdorff des $\tilde{f}_{n}(\partial D)$; alors (5.2) montre que $\tilde{f}_{\infty}$ n'est pas une application constante.

Montrons que $\tilde{f}_{\infty}$ est une immersion. Considérons pour cela la suite des relevés $\tilde{f}_{n}$ des $\tilde{f}_{n}$ dans la grassmannienne $G_{2} V$ des 2-plans complexes de $V$. C'est un fibré sur $E$ dont la fibre, conforme à $C P^{1}$, est kählerienne. De plus, le relevé dans $G_{2} V$ d'une immersion pseudo-holomorphe à valeurs dans $E$ est presque-complexe car c'est la composée du 1-jet de l'application (dont il est connu qu'elle est pseudo-holomorphe à valeurs dans un fibré presque-complexe, cf. l'appendice de [Pan]) par la projection sur $G_{2} V$, qui est une application presque-complexe (il suffit pour le montrer de vérifier que c'est vrai dans la fibre, et donc dans le cas où on remplace $V$ par $C^{2}$ ). De plus, les $\overline{\tilde{f}_{n}}$ sont en fait à valeur dans l'ensemble des 2-plans de $V$ qui se projettent sur TS avec un déterminant positif, qui est en chaque point de $E$ une hémisphère. Si on se limite à la convergence au voisinage d'un compact de l'image de $\tilde{f}_{\infty}$, on se trouve donc dans les conditions de la proposition 3.8, avec pour compact $K$ le produit de cet hémisphère (compact et homéomorphe à $D^{2}$ ) avec un voisinage compact de l'image de $\tilde{f}_{\infty}$ dans $E$. La proposition 3.8 nous fournit une majoration des dérivées des $\tilde{f}_{n}$, et on peut donc a nouveau appliquer le théorème d'Ascoli et se ramener à une sous-suite de $\tilde{\tilde{f}}_{n}$ qui converge ; la sous-suite associée de $\left(\tilde{f}_{n}\right)$ converge alors d'après 3.6 vers une immersion pseudo-holomorphe, et $\tilde{f}_{\infty}$ est donc une immersion.

Nous avons donc montré que les $\tilde{f}_{n}$ convergent $C^{\infty}$ vers une immersion pseudo-holomorphe de $D$ dans $E$. Or les $W_{n}$ peuvent s'interpreter comme les déterminants des projections des $j^{1} f_{n}(S)$ sur $S$, et ils convergent donc $C^{\infty}$ vers le déterminant de la projection de l'image des $\tilde{f}_{n}$ sur $S$, soit $W$. Ce résultat correspond d'ailleurs aussi à l'ellipticité des équations considérées !

Il reste à utiliser des majorations issues de la proposition 5.1 pour se ramener au principe du maximum pour un opérateur elliptique (visible dans la proposition 5.1) qui nous montre que si $W=0$ en un point, alors $W=0$ dans un voisinage de ce point, et donc $W=0$ partout. 
On se restreint ensuite à la sous-suite de $\left(f_{n}\right)$ associée à une sous-suite de $\left(W_{n}\right)$ qui converge; on la notera encore $\left(f_{n}\right)$. On peut alors étudier ce qui se passe dans les deux cas; d'abord

Lemme 5.3. Si $W_{\infty}$ n'est jamais nulle, alors $\tilde{f}_{\infty}(D)$ est inclus dans le graphe du 1-jet d'une immersion isométrique $f_{\infty}$, et $\left(f_{n}\right)_{n \in N}$ converge $C^{\infty}$ vers $f_{\infty}$.

Démonstration. (Cf. [Lab89]) $W$ est le déterminant de la projection de $\tilde{f}_{\infty}(D)$ sur $S$, donc dans ce cas $\tilde{f}_{\infty}(D)$ est inclus dans le graphe du 1-jet d'une immersion isométrique. Le lemme 2.2.3 implique alors la régularité de la convergence.

On peut décrire ce qui se passe localement dans le cas d'une dégénérescence; notons pour cela $B$ l'application linéaire symétrique associée à la deuxième forme fondamentale, c'est à dire que pour $v, w \in T_{x} S, I(v, w)=$ $I(v, B w)$. On peut alors énoncer le

Lemme 5.4. Si $W_{\infty}$ est toujours nul, alors $\tilde{f}_{\infty}(D)$ est inclus dans une surface de pli $\Sigma_{(\gamma, \Gamma)}$; de plus, pour $\alpha$ assez petit, $\left.\gamma\right|_{[-\alpha, \alpha]}$ est limite des courbes intégrales $c_{n}$ passant par $\gamma(0)$ de la direction principale associée à la plus petite valeur propre de $B_{n}$. De même, $\Gamma$ est la limite des $f_{n}\left(\dot{c}_{n}\right)$. Enfin, $\tilde{f}_{\infty}$ est une isométrie locale de $\tilde{\gamma}$ sur $\Gamma$.

Démonstration. (Cf. [Lab89]) Dans ce cas, $\left(W_{n}\right)_{n \in N}$ converge $C^{\infty}$ vers 0 car $W$ est le déterminant de la projection de $\left(j^{1} f\right)(S)$ sur $S$. Donc d'après la première équation de la proposition $5.1, \pi_{S}^{*} \omega$ converge aussi vers $0 . \mathrm{Si}$ on note $c_{n}$ la courbe intégrale de la direction associée à la plus petite valeur propre de $B$, sa courbure géodésique tend donc vers 0 et $c_{n}$ tend vers une géodésique $\gamma$ de $S$, et comme la valeur propre correspondante de $B$ tend vers 0 sur $\gamma, h_{n}\left(c_{n}\right)$ tend vers une géodésique $\Gamma$ de $M$. On en déduit que $\tilde{f}_{\infty}(D)$ a une infinité de points communs avec la "surface de pli" $\Sigma_{(\gamma, \Gamma)}$ et donc (principe de Carleman pour deux courbes pseudo-holomorphes, cf [McD91]) $\tilde{f}_{\infty}(D) \subset \Sigma_{(\gamma, \Gamma)}$.

Il faut remarquer que l'aire, pour la métrique induite par celle de $E$, du domaine d'une surface de pli $\Sigma_{(\gamma, \Gamma)}$ qui se projette sur un segment $\gamma_{\mid\left[t_{0}, t_{1}\right]}$ donné est infinie; comme la convergence vers les surfaces de pli est uniforme sur les compacts, on va voir que le fait que $W_{\infty}=0$ implique (en revenant 
Surfaces convexes dans des espaces lorentziens à courbure constante 309

sur $\left(S, g_{\infty}\right)$ ) la divergence de l'intégrale de la courbure moyenne (qui correspond à l'aire sur $j^{1} f(S)$ pour la métrique induite par celle de $E$ ) dans tout voisinage d'un point du pli. Une hypothèse de borne uniforme de cette quantité pour les $f_{n}$ conduit donc à exclure la convergence possible de $\left(j^{1} f_{n}\right)_{n \in N}$ vers une surface de pli, et on a le:

Théorème 5.5. Soit $\phi_{n}: D \rightarrow M$ une suite d'immersions de type espace uniformément elliptiques du disque telles que $\left(\phi^{*} g_{0}\right)_{n \in N}$ converge $C^{\infty}$ vers une métrique $g_{\infty}$ et que la suite des intégrales des courbures moyennes $H_{n}$ soit majorée par une constante, et soit $\left(x_{n}\right)_{n \in N}$ telle que $\left(x_{n}\right)$ et $\left(j^{1} \phi_{n}\left(x_{n}\right)\right)$ convergent. Alors il existe une suite extraite de $\phi_{n}$ qui converge $C^{\infty}$ vers une immersion isométrique.

Démonstration. Soit $K_{p}$ une suite de compacts de $D$ contenant $\lim _{n \rightarrow \infty} x_{n}$.

Supposons qu'il existe un $p$ tel que la courbure moyenne $H_{n}$ des $\phi_{n}$ ne soit pas bornée sur $K_{p}$. Par compacité de $K_{p}$, on pourrait trouver une suite extraite des $\phi_{n}$ et une suite $\left(y_{m}\right)_{m \in N}$ de points de $D$ convergeant vers $y_{\infty}$ telles que $H_{m}\left(y_{m}\right)$ tende vers l'infini, et on aurait alors (éventuellement en remplaçant les $f_{n}$ par des $k_{n} \circ f_{n}$, où $\left.k_{n} \in \operatorname{Isom}(M)\right)$ apparition d'un "pli" $\Sigma_{(\gamma, \Gamma)}$ en $y_{\infty}$ à la limite.

L'aire de la région $\Omega$ de $\Sigma_{(\gamma, \Gamma)}$ qui se trouve au-dessus d'un segment $\gamma_{[[r, s]}$ suffisamment petit de $\gamma$ contenant $y_{\infty}$ est infinie; or toute cette région est contenue dans la limite $L$ de $\left(\phi_{n}(D)\right)_{n \in N}$, sans quoi il existerait un $z \in \Omega \cap \partial L$; on pourrait alors trouver une suite $\left(t_{n}\right)$ d'éléments de $D$ tels que $\left(j^{1} \phi_{n}\left(t_{n}\right)\right)$ converge vers $z$, ce qui impliqueraït que $\left(t_{n}\right)$ converge vers un point du pli. Mais alors d'après la proposition 4.6, on en déduirait qu'il existe $A, B>0$ et une suite de voisinages $U_{n}$ des $t_{n}$ dans $D$, homéomorphes au disque, tels que:

- $\int_{U} H \leq A$

- $d_{E}\left(z, j^{1} f_{n}\left(\partial U_{n}\right)\right) \geq B$

et une boule de centre $z$ et de rayon $B$ serait dans $L$, donc $z$ ne pourrait donc pas être sur le bord de $L$.

Il faut alors remarquer que l'aire de $j^{1} \phi_{n}(D)$ est égale à l'intégrale sur $D$ de $H_{n}$, qui est bornée par hypothèse. L'existence d'un $p$ tel que la courbure des $\phi_{n}$ ne soit pas bornée entraîne donc une contradiction, et donc pour tout $p$, il existe une borne sur les fonctions $H_{n}$ sur $K_{p}$.

Or une borne sur les $H_{n}$ permet de contrôler le rapport des éléments d'aire pour $\sigma$ et pour $I I$, ainsi que le facteur conforme entre $I$ et la métrique 
induite par les $j^{1} \phi_{n}$ sur $S$; donc si les $H_{n}$ sont bornés sur $K_{p}$, et puisque $\left(j^{1} \phi_{n}\left(x_{n}\right)\right)$ converge, $\phi_{n}\left(K_{p}\right)$ reste inclus dans un compact de $E$. On peut donc (par un procédé diagonal) trouver une suite extraite de $\left(j^{1} \phi_{n}\right)$ qui converge sur une suite de points dense dans $K_{p}$, et donc sur $K_{p}$. On obtient ainsi une sous-suite de $\left(\phi_{n}\right)$ qui converge $C^{0}$ sur $D$, et on peut appliquer les lemmes 5.2 et 5.4 pour conclure.

Contrairement à ce qui se passe dans le cas riemannien, des dégénérescences d'immersions de surfaces compactes peuvent réellement se produire; le lemme ci-dessus nous donne alors une indication précise de la façon dont les choses se passent. En effet, supposons que les images $S_{n}$ des immersions $f_{n}: S \rightarrow M$ convergent $C^{0}$ vers $S_{\infty}$ mais ne convergent pas $C^{1}$ en $s \in$ $S$, alors que $\left(j^{1} f_{n}(s)\right)_{n \in N^{*}}$ converge, et que les $f^{*} g_{0}$ convergent $C^{\infty}$ vers une métrique $\sigma$ riemannienne; les images des applications $\phi_{n}$ associées dans l'espace des 1-jets convergent (sur les compacts) vers une surface de pli, et les $S_{n}$ convergent donc vers une surface pliée le long d'une géodésique $\gamma$, et tangente au cône de lumière en dehors de $\gamma$. Cela entraîne que $\sigma$ admet une géodésique fermée passant par $f_{\infty}(s)$, et de longueur $2 \pi$ puisque elle coïncide avec une géodésique de $M$. C'est grâce à cette remarque qu'on va pouvoir "éviter" les dégénérescences dans la suite pour trouver un résultat d'existence d'immersions.

Théorème 5.6. Soit $\left(f_{n}\right)_{n \in N}: D \rightarrow M$ une suite d'immersions de type espace uniformément elliptiques du disque, telle que $\left(f_{n}^{*} g_{0}\right)_{n \in N}$ converge $C^{\infty}$ vers $g_{\infty}$. Soit $\left(x_{n}\right)_{n \in N}$ une suite convergeant vers $x_{\infty}$ telle que $\left(j^{1} f_{n}\left(x_{n}\right)\right)$ converge, mais sans que $\left(f_{n}\right)$ converge $C^{\infty}$ au voisinage de $x_{\infty}$. Alors il existe une suite extraite de $\left(f_{n}\right)_{n \in N}$, qu'on notera encore $\left(f_{n}\right)_{n \in N}$, une géodésique maximale $\gamma \ni x_{\infty}$ de $\left(D, g_{\infty}\right)$, et un segment géodésique $\Gamma$ de $M$, tels que $\left(f_{n \mid \gamma}\right)$ converge vers une isométrie sur $\Gamma$.

Démonstration. On peut appliquer la proposition 4.6 pour se ramener dans la situation décrite après la proposition 5.1 ; les "bons voisinages" que cette proposition nous donne permettent en effet (grâce à la représentation conforme) de faire apparaître des applications de $D$ dans $E$ pseudo-holomorphes auxquelles on peut appliquer le lemme 2.2.3.

On peut alors appliquer un raisonnement similaire a celui qu'on a fait dans la démonstration du théorème 5.5 , et extraire de $\left(j^{1} f_{n}\right)$ une sous-suite convergeant $C^{\infty}$ vers sur les compacts vers une courbe pseudo-holomorphe. 
On a supposé que $\left(f_{n}\right)$ ne converge pas $C^{\infty}$ au voisinage de $x$, ce qui implique que $H$ y devient infinie (sans quoi le théorème 5.5 s'appliquerait). Mais alors grâce à la proposition $4.3, W_{\infty}$ est toujours nul, et grâce au lemme 5.4 , on sait que $\left(j^{1} f_{n}\right)(D)$ converge vers une surface de pli, ce qui implique en revenant de $E$ dans $M$ qu'il existe un $\gamma$ correspondant au pli, et que $f_{\infty}$ est une isométrie de $\gamma$ sur un segment géodésique $\Gamma$ de $M$ (cf. lemme 5.4).

Or si la suite des $j^{1} f_{n}(D)$ tend vers une surface de pli sur un compact, elle y converge au-dessus de $D$ tout entier (principe de Carleman a nouveau), ce qui se traduit par le fait que $\gamma$ peut se prolonger jusqu'au bord de $D$. De même, il ne peut pas y avoir 2 telles géodésiques $\gamma_{1}$ et $\gamma_{2}$, sans quoi $j^{1} f_{n}(D)$ convergerait $C^{\infty}$ vers $\Sigma_{\left(\gamma_{1}, \Gamma_{1}\right)}$ sur un compact et vers $\Sigma_{\left(\gamma_{2}, \Gamma_{2}\right)}$ sur un autre; mais alors $j^{1} f_{n}$ convergerait (par connexité de $D$ ) vers $\Sigma_{\left(\gamma_{1}, \Gamma_{1}\right)}$ et vers $\Sigma_{\left(\gamma_{2}, \Gamma_{2}\right)}$ sur tous les compacts (cf. le principe de Carleman), et on devrait avoir que $\Sigma_{\left(\gamma_{1}, \Gamma_{1}\right)}=\Sigma_{\left(\gamma_{2}, \Gamma_{2}\right)}$, ce qui impliquerait que $\gamma_{1}=\gamma_{2}$.

En fait, la convergence se fait, au voisinage d'un point de l'intérieur de $\Gamma$ et en dehors de $\Gamma$, vers une sur surface immergée tangente au cône de lumière, puisque on a convergence vers $\Sigma_{(\gamma, \Gamma)}$ dans les compacts de $E$. Cette situation est d'ailleurs assez différente de celle qui se présente dans le cas riemannien, ou F. Labourie a montré [Lab87] qu'il y a "enroulement" des 1jets des immersions isométriques autour des surfaces de plis, qui apparaîssent comme des "tubes".

Remarque. Il existe une réciproque: si une telle géodésique $\gamma$ existe et est envoyée sur une géodésique de $M$, alors il n'y a convergence $C^{\infty}$ en aucun point de $\gamma$. En effet, si il y avait convergence $C^{\infty}$ ce serait vers une immersion isométrique (proposition 5.2 et lemme 5.3); or dans ce cas l'une des courbures principales serait nécessairement nulle sur $\gamma$ (car son image étant géodésique, on aurait $I\left(\gamma^{\prime}, \gamma^{\prime}\right)=0$ alors que $I$ est une forme positive de déterminant non nul dans le cas elliptique), et la courbure moyenne serait donc infinie, ce qui est impossible pour une immersion isométrique.

Corollaire 5.7. Soit $\left(f_{n}\right)_{n \in N}$ une suite d'immersions de $S^{2}$ dans $S_{1}^{3}$, telle que $\left(\sigma_{n}\right)_{n \in N}=\left(f_{n}^{*} g_{0}\right)_{n \in N}$ soit une suite de métriques sur $S^{2}$ convergeant $C^{\infty}$ vers une métrique $\sigma_{\infty}$ vérifiant la condition suivante:

sa courbure vérifie $K_{\sigma_{\infty}} \leq 1-\epsilon$ et $\sigma_{\infty}$ n'admet aucune géodésique fermée de longueur $L \leq 2 \pi$.

Supposons de plus qu'il existe une suite $\left(x_{n}\right)_{n \in N}$ de points de $S^{2}$ qui converge vers $x_{\infty}$ et telle que $j^{1} f_{n}\left(x_{n}\right)$ converge dans $E$. Alors il existe une suite 
extraite de $\left(f_{n}\right)_{n \in N}$ qui converge $C^{\infty}$ vers une immersion isométrique $f_{\infty}$ de $\left(S^{2}, \sigma_{\infty}\right)$ dans $S_{1}^{3}$.

Démonstration. C'est une conséquence du théorème 5.6, car nos hypothèses d'une part impliquent l'uniforme ellipticité de la suite d'immersions, et d'autre part empêchent la dégénérescence en interdisant l'apparition d'un pli le long d'une géodésique de longueur $2 \pi$. Or toutes les géodésiques fermées de $S_{1}^{3}$ sont de longueur $2 \pi$, car l'une d'entre elle a longueur $2 \pi$ (cf. le modèle de $S_{1}^{3}$ qui se trouve dans l'introduction) et les autres s'en déduisent par action du groupe d'isométries de $S_{1}^{3}$, qui agit transitivement sur $T S_{1}^{3}$. On peut donc appliquer les mêmes méthodes que pour la démonstration du théorème 5.5 pour montrer qu'il existe une suite extraite de $\left(j^{1} f_{n}\right)$ qui converge $C^{0}$, puis la proposition 5.2 et les lemmes 5.3 et 5.4 pour en déduire que la suite correspondante extraite de $\left(f_{n}\right)_{n \in N}$ converge vers une immersion isométrique, car les plis sont interdits.

C'est cet énoncé qui va nous permettre de donner des résultats d'existence et d'unicité d'immersions isométriques elliptiques; il décrit en effet les dégénéréscences de façon assez précise pour permettre de montrer que dans certains cas, elles ne peuvent pas se produire, ce qui reviendra a savoir qu'un certain opérateur est propre.

\section{Analyse.}

Le contenu de cette section est plus ou moins classique, les démonstrations seront donc raccourcies. Le lecteur pourra se reporter à [Ham82] pour les outils fondamentaux, ou à [Lab89] où la même mécanique est utilisée dans un cas similaire.

Le moteur "réel" des opérations qui sont effectuées est l'ellipticité des équations qui décrivent notre système, mais il est ici "caché" derrière les courbes pseudo-holomorphes et en particulier le lemme de Schwarz.

Définition 6.1. On choisit un point $s$ de $S^{2}$ et un $\epsilon>0$; on notera dans la suite:

- $F$ l'espace des immersions $f: S^{2} \rightarrow S_{1}^{3}$ telle que $f^{*} g_{S_{1}^{3}}$ soit à courbure $K<1-\epsilon$, muni de la topologie $C^{\infty}$;

- $G$ l'espace des métriques sur $S^{2}$ à courbure $K<1$ et dont toutes les géodésiques fermées sont de longueur strictement supérieure à $2 \pi$, muni de la topologie $C^{\infty}$; 
- $H$ l'espace des couples $\left(g_{0}, I\right)$ où $g_{0} \in G$ et $I$ est une isométrie de $\left(T_{s} S^{2}, g_{0}\right)$ dans $T S_{1}^{3}$, muni de la topologie "produit" naturelle;

- $P$ l'opérateur de $F$ dans $H$ qui à une immersion $f$ associe le couple $\left(f^{*} g_{0}, T_{s} f\right)$.

Lemme 6.2. $P$ est un revêtement à deux feuillets.

Démonstration. Elle découle immédiatement des propositions 6.3 à 6.6 .

Proposition 6.3. $H$ est connexe.

Démonstration. Il suffit de montrer que $G$ est connexe; en fait il est facile de voir qu'il est même connexe par arcs. Soit en effet $\sigma_{1}, \sigma_{2} \in G$; posons $\sigma_{t}=t \sigma_{1}+(1-t) \sigma_{2}$. $\sigma_{t}$ est une métrique riemannienne pour tout $t \in[0,1]$; par ailleurs, notons $K$ la borne supérieure des courbures, $L$ la borne inférieure des longueurs des géodésiques fermées des $\sigma_{t}$, et $M=\max \left(K /(1-\epsilon),(L / 2 \pi)^{2}\right)$. Définissons $\sigma_{t}^{\prime}$ pour $t \in[0,1]$ par

- si $t \in\left[0, \frac{1}{3}\right], \sigma_{t}^{\prime}=\left(1-3 t+\frac{3 t}{M}\right) \sigma_{1}$;

- si $t \in\left[\frac{1}{3}, \frac{2}{3}\right], \sigma_{t}^{\prime}=\frac{1}{M} \sigma_{3 t}$;

- si $t \in\left[\frac{2}{3}, 1\right], \sigma_{t}^{\prime}=\left(3 t-2+\frac{3-3 t}{M}\right) \sigma_{2}$;

Il est clair par construction que pour tout $t \in[0,1], \sigma_{t}^{\prime} \in G$.

Proposition 6.4. $P$ est un homéomorphisme local.

Démonstration. Voir [Lab89]; il faut vérifier qu'on est dans le cadre des bons espaces de Fréchet développé dans [Ham82], ce qui correspond à l'ellipticité des équations, et utiliser le théorème de Nash-Moser.

Proposition 6.5. $P$ est propre.

Démonstration. C'est une conséquence du corollaire 5.7. En effet, supposons que $\left(f_{n}\right)_{n \in N}, f_{n} \in F$, est telle que $\left(j^{1} f_{n}(s)\right)$ converge et que les $\left(f_{n}^{*} g_{0}\right)$ convergent $C^{\infty}$. On se trouve (grâce à la définition de $G$ ) dans la situation du corollaire 5.7 , avec une suite $\left(x_{n}\right)_{n \in N}$ constante égale à $s$, et il existe donc 
une suite extraite de $\left(f_{n}\right)$ qui converge $C^{\infty}$ vers une immersion isométrique de $\left(S^{2}, \sigma_{\infty}\right)$ dans $S_{1}^{3}$. Donc $\left(f_{n}\right)$ converge dans $F$. Nous avons donc montré que si une suite $\left(h_{n}\right)_{n \in N}$ d'éléments de $H$ est telle que $\left(P\left(h_{n}\right)\right)$ converge, alors $\left(h_{n}\right)$ admet une sous-suite convergente, ce qui implique que $P$ est propre.

Il reste à trouver un cas simplement analysable pour servir de "germe" à l'argument de déformation qui se trouve plus haut; le plus simple est de se servir de $S^{2}$ munie de la métrique à courbure constante égale à $\left.\epsilon \in\right] 0,1[$; on notera cette métrique $\sigma_{\epsilon}$. On a le

Lemme 6.6. Si $\epsilon \in] 0,1\left[, x_{0} \in S^{2}, y_{0} \in S_{1}^{3}\right.$, et $g \in \operatorname{Isom}\left(T_{x_{0}} S^{2}, T_{y_{0}} S_{1}^{3}\right)$, alors il existe exactement deux immersions isométriques de $\left(S^{2}, \sigma_{\epsilon}\right)$ dans $S_{1}^{3}$ admettant $g$ comme 1 -jet en $x_{0}$, et elles sont totalement ombilicales.

Démonstration. plutôt que d'utiliser une version élémentaire du principe du maximum pour la courbure moyenne, on peut faire appel à la dualité entre $S_{1}^{3}$ et $H^{3}$. En effet, il existe exactement deux immersions isométriques de $\left(S^{2}, \sigma_{\frac{\epsilon}{1-\epsilon}}\right)$ dans $H^{3}$ ayant un 1-jet donné en un point donné (cf. [Lab89] ou [Pog73]), et on en déduit en passant par les modèles canoniques de $S_{1}^{3}$ et de $H^{3}$ dans $R_{1}^{3}$ qu'il existe exactement deux immersions isométriques de $\left(S^{2}, \sigma_{\epsilon}\right)$ dans $S_{1}^{3}$ ayant un 1-jet fixé en un point (cf. l'introduction). Réciproquement, si $\phi$ est une immersion isométrique de $\left(S^{2}, \sigma_{\epsilon}\right)$ dans $S_{1}^{3}$, on en déduit par dualité une immersion isométrique de $\tilde{\phi}$ de $\left(S^{2}, \sigma_{\frac{\epsilon}{1-\epsilon}}\right)$ dans $H^{3}$; or il existe exactement deux telles immersions (si on fixe le 1 -jet en un point, cf. à nouveau [Lab89] ou [Pog73]) et elles sont totalement ombiliques ; on en déduit que $\phi$ est totalement ombilique car les courbures moyennes $H$ et $\tilde{H}$ de $\phi$ et $\tilde{\phi}$ sont reliées par

$$
\tilde{H}=\frac{H}{1-\epsilon}
$$

En fait, les deux immersions isométriques correspondent aux deux cotés du plan tangent à la surface immergée au point où on fixe le 1-jet : on peut décider que la surface se trouve d'un coté ou de l'autre de son plan tangent, et on a alors existence et unicité des immersions isométriques à 1-jet donné.

Corollaire 6.7. Chaque point de $H$ a exactement deux images réciproques par $P$. 
Démonstration. C'est clair car le nombre d'images réciproques de $P$ est constant sur $H$ d'après les propriétés de $P$ ci-dessus.

On déduit des résultats ci-dessus le fait plus géométrique suivant:

Proposition 6.8. Soit $\sigma$ une métrique sur $S^{2}$ à courbure $K<1$ et sans géodésique de longueur $L \leq 2 \pi, x_{0} \in S^{2}, y_{0} \in S_{1}^{3}$, et $I \in \operatorname{Isom}\left(T_{x_{o}} S^{2}, T_{y_{0}} S_{1}^{3}\right)$. Alors il existe exactement deux immersions isométriques $\phi$ de $\left(S^{2}, \sigma\right)$ dans $S_{1}^{3}$ telle que $j^{1} \phi\left(x_{0}\right)=I$.

Démonstration. $P$ est un revêtement à deux feuillets, donc en particulier chaque élément de l'ensemble d'arrivée a exactement deux images réciproques.

Bien entendu, les remarques faites dans l'introduction montrent que ces immersions sont en fait des plongements! De plus, elles se déduisent l'une de l'autre par une isométrie globale de $S_{1}^{3}$.

Il faut noter que l'argument de déformation que nous avons utilisé ne permet absolument pas d'obtenir des informations sur les immersions isométriques de métriques qui admettent des géodésiques de longueur $L \leq$ $2 \pi$. En effet, la fonction qui à une métrique sur $S^{2}$ associe la longueur de sa plus courte géodésique fermée est continue ; donc tout chemin joignant une métrique dont les géodésiques fermées sont de longueur $L>2 \pi$ à une autre ayant une géodésique fermée de longueur $L \leq 2 \pi$ passe nécessairement par une métrique admettant une géodésique de longueur exactement $2 \pi$, et une dégénérescence du chemin associé dans l'espace des immersions isométriques peuvent se produire. La section suivante montre même qu'une telle dégénérescence doit nécessairement se produire, sans quoi on obtiendrait une immersion isométrique que les résultats qui suivent interdisent.

\section{Réciproque.}

Il est naturel de se demander si la proposition 5.8 est optimale, dans le sens où les conditions qu'elle pose pour l'existence d'une immersion isométrique elliptique d'une surface dans $S_{1}^{3}$ sont vraiment nécessaires, et en particulier si la condition concernant les longueurs des géodésiques fermées est naturelle. La réponse (positive) est donnée par le lemme suivant et par son corollaire: 
Lemme 7.1. Soit $\gamma: R / L Z \rightarrow S_{1}^{n}$ une courbe $C^{2}$ telle que

$$
\forall t \in[0, L],\left\langle\gamma^{\prime}(t) \mid \gamma^{\prime}(t)\right\rangle=1 \text { et }\left\langle\gamma^{\prime \prime}(t) \mid \gamma^{\prime \prime}(t)\right\rangle<0
$$

Alors $L>2 \pi$.

Corollaire 7.2. $S i$ une surface $(S, \sigma)$ admet une immersion isométrique elliptique dans $S_{1}^{3}$, alors ses géodésiques fermées sont de longueur $L>2 \pi$.

Démonstration. (du corollaire 7.2) Le corollaire est évident si on admet le lemme, car si $\gamma$ est une géodésique (paramétrée par la longueur d'arc) tracée sur une surface immergée elliptiquement dans $S_{1}^{3}$, alors son accélération est partout strictement de type temps.

Un analogue de ce corollaire se trouve dans [RH93] dans le cas où $S$ est un polygone de $S_{1}^{3}$ (dont les faces sont totalement géodésiques et les arêtes géodésiques). $\gamma$ est alors une courbe polygonales fermée. La preuve de [RH93] repose sur la dualité de $S_{1}^{3}$ avec $H^{3}$, et ne se généralise donc pas immédiatement au cas de $S_{1}^{n}$. Une version polyhèdrale du lemme 7.1 a été trouvée (indépendamment) par R. Charney et M. Davis (voir [CD94]) mais, là aussi, la démonstration est très différente de celle qui suit.

Démonstration. (du lemme 7.1) Nous allons procéder en plusieurs étapes en nous ramenant à un problème très simple d'étude de courbes intégrales d'un champ de vecteur.

- Notons $\gamma(t)=(\sigma(t), r(t))$ avec $\sigma(t) \in S^{n}, r(t) \in R$, dans un modèle de $S_{1}^{n} \simeq\left(S^{n} \times R, \operatorname{ch}(r)^{2} \operatorname{can}_{S^{n}}-d r^{2}\right)$; choisir ce modèle revient à choisir la sphère $S^{n}$ totalement géodésique qui y correspond à l'équation $r=0$, ainsi que l'orientation de son vecteur normal. On vérifie à la main que $\gamma^{\prime \prime}=\nabla_{\gamma^{\prime}} \gamma^{\prime}$ s'exprime sous la forme :

$$
\gamma^{\prime \prime}(t)=\left(r^{\prime \prime}+\operatorname{sh}(r) \cdot \operatorname{ch}(r)\left\|\sigma^{\prime}\right\|^{2}\right) \frac{d}{d r}+\left(\partial_{t}\left\|\sigma^{\prime}\right\|+\operatorname{th}(r)\left\|\sigma^{\prime}\right\| \cdot r^{\prime}\right) u+\sigma^{\prime \prime}
$$

et donc que la la condition " $\left\langle\gamma^{\prime \prime}(t) \mid \gamma^{\prime \prime}(t)\right\rangle<0$ " se traduit par l'inégalité:

$$
\left[r^{\prime \prime}+\operatorname{sh}(r) \cdot \operatorname{ch}(r)\left\|\sigma^{\prime}\right\|^{2}\right]^{2}>\left[\partial_{t}\left\|\sigma^{\prime}\right\|+\operatorname{th}(r)\left\|\sigma^{\prime}\right\| \cdot r^{\prime}\right]^{2}+\left\|\sigma^{\prime \prime}\right\|^{2}
$$

Dans le cas où $\left\langle\gamma^{\prime} \mid \gamma^{\prime}\right\rangle=1,\left\|\sigma^{\prime}\right\|$ dépend simplement de $r^{\prime}$ et $\partial_{t}\left\|\sigma^{\prime}\right\|$ de $r^{\prime}$ et de $r^{\prime \prime}$, donc en négligeant le terme en $\left\|\sigma^{\prime \prime}\right\|$ et en isolant ceux en 
Surfaces convexes dans des espaces lorentziens à courbure constante 317 $r^{\prime \prime}$, on obtient que

$$
r^{\prime \prime}>\Phi\left(r, r^{\prime}\right)
$$

avec $\Phi(x, y)<0$ dans le domaine $R_{+}^{*} \times R_{-}^{*} \subset R^{2}$ (ou que $r^{\prime \prime}<\Psi\left(r, r^{\prime}\right)$ dans le cas où la convexité de $\gamma$ est supposée dirigée vers les $r$ décroissants, ce qui ne change rien).

- Supposons qu'il existe $s<t$ tels que $r(s)>0, r^{\prime}(s)=0, r(t)=0$, et $r^{\prime}<0$ dans $\left.] s, t\right]$; on associe à $\gamma_{[[s, t]}$ une courbe $(x(\tau), y(\tau)) \in R^{2}$ en posant $x(\tau)=r(\tau), y(\tau)=r^{\prime}(\tau) ;$ comme $\left\langle\gamma^{\prime} \mid \gamma^{\prime}\right\rangle=1$, on a

$$
\mathrm{L}\left(\gamma_{\mid[s, t]}\right)=\int_{s}^{t} d \tau=\int_{s}^{t}\left(\frac{\partial x}{\partial \tau}\right)^{-1} d x=\int_{s}^{t} \frac{d x}{y}
$$

et $(x, y)$ est telle que

$$
x^{\prime}(\tau)=y(\tau), y^{\prime}(\tau)>\Phi(x(\tau), y(\tau))
$$

- Il existe une unique courbe intégrale $\left\{\left(x_{0}(\tau), y_{0}(\tau)\right) \mid \tau \in\left[r_{0}, s_{0}\right]\right\}$ du champ de vecteurs $X$ de coordonnées $(y, \Phi(x, y))$ sur $R^{2}$ partant de $(x(r), 0)$ et allant jusqu'à l'axe $O y$; elle correspond à un segment de géodésique $\gamma_{0}$ de $S_{1}^{n}$ (c'est à dire au cas où $\sigma^{\prime \prime} \equiv 0$ dans 7.1) allant d'un point où sa valeur de $r$ est maximale à un point où elle est nulle, et sa longueur est donc $\frac{\pi}{2}$ (il s'agit d'un quart de géodésique de $S_{1}^{n}$, et ces géodésiques ont toutes longueur $2 \pi$ car elles se déduisent toutes les unes des autres par des isométries de $S_{1}^{n}$ ).

- $(x(\tau), y(\tau))$ et $\left(x_{0}(\tau), y_{0}(\tau)\right)$ ne peuvent pas avoir de point commun à cause de 7.2, et $\gamma$ est donc toute entière située dans le domaine de $R^{2}$ borné par $O x, O y$ et $\gamma_{0}$ et ainsi, quand $\left.\left.\tau \in\right] r, s\right]$ et $x(\tau)=x_{0}(\tau)$, on a $y(\tau)>y_{0}(\tau)$.

- de plus, $x^{\prime}(\tau)=y(\tau)<0$ sur $\left.] r, s\right]$, donc on a

$$
\int_{r}^{s} \frac{d x}{y}>\int_{r_{0}}^{s_{0}} \frac{d x_{0}}{y_{0}}
$$

et donc $\mathrm{L}\left(\gamma_{\mid[r, s]}\right)>\mathrm{L}\left(\gamma_{0 \mid\left[r_{0}, s_{0}\right]}\right)=\frac{\pi}{2}$

- En répétant l'opération pour $t<s, r(t)=0, r^{\prime}(s)=0, r^{\prime}>0$ sur $[t, s[$, on obtient que si $r(s)=r(t)=0$ et $r>0$ sur $] s, t\left[\right.$, alors $\gamma_{\mid[s, t]}$ est de longueur strictement supérieure à $\pi$. Il suffira donc pour conclure 
de choisir un modèle de $S_{1}^{n}$ dans lequel la fonction $r$ associée à $\gamma$ soit strictement positive sur deux intervalles ouverts disjoints de $R / L Z$, et donc de trouver une sphère totalement géodésique $S^{n}$ dans $S_{1}^{n}$ que $\gamma$ coupe au moins 3 fois.

- Une telle sphère existe: choisissons en effet un (n-1)-plan $P \ni \gamma^{\prime}(0)$ de type espace de $T_{\gamma(0)} S_{1}^{n}$, et notons $\left(S_{t}\right)_{t \in R}$ la famille des $S^{n}$ totalement géodésiques dont le tangent contient $P$, indicées par l'angle qu'elle font avec l'une d'entre elle notée $S_{0}$. Soit $I$ l'ensemble des $u \in R$ tels que $S_{u}$ rencontre $\gamma$ en-dehors de $\gamma(0)$. I ne peut être réduit à un point, sans quoi $\gamma$ serait incluse dans une hypersurface totalement géodésique de type espace et ne pourrait pas être elliptique; donc $I$ contient un intervalle, et par une application directe du lemme de Sard, on voit qu'il existe $u_{0} \in R$ telle que $S_{u_{0}}$ est transversale à $\gamma$. Il existe alors deux ouverts ] $s, 0$ [ et ]0,t de $R / L Z$ sur lesquels le $r$ associé à $\gamma$ dans le modèle de $S_{1}^{n}$ dans lequel $S_{u_{0}}=\{r=0\}$ est positif. On clôt ainsi la démonstration.

Pour rendre la démonstration qui précède un peu plus transparente, la remarque suivante (due à François Labourie) est importante : le cas où $n>2$ se déduit simplement du cas $n=2$, ou plutôt du fait que si, dans le revêtement universel $\tilde{S}_{1}^{2}$ de $S_{1}^{2}$, une courbe elliptique $\gamma$ rencontre pour $t=0$ une géodésique $g_{0}\left(g_{0}^{\prime}\right.$ étant alors dirigée dans la direction opposée à la convexité de $\gamma$ ) alors $\gamma(t) \notin g_{0}$ pour $t \leq \pi$. En effet, on en déduit le résultat sur les intersections d'une courbe elliptique $\gamma$ avec une hypersurface totalement géodésique $H$ car la surface engendrée dans $S_{1}^{n}$ par les normales à $H$ passant par $\gamma$ est à courbure de Gauss nulle, donc est un quotient de $\tilde{S}_{1}^{2}$, ce qui implique que entre deux intersections avec $H$, la longueur de $\gamma$ est au moins $\pi$ quand sa convexité est dans le bon sens. Par contre, le résultat dans $\tilde{S}_{1}^{2}$ doit se démontrer essentiellement comme plus haut dans le cas de $S_{1}^{n}$.

Le corollaire 7.2 nous montre à posteriori que des dégénérescences de suites d'immersions de sphères dans $S_{1}^{3}$ peuvent vraiment se produire; sinon, les raisonnements faits plus hauts seraient encore valables pour des $(S, \sigma)$ admettant des géodésiques de longueur $L \leq 2 \pi$, et on pourrait en déduire un résultat d'existence d'immersion isométrique de telles surfaces dans $S_{1}^{3}$, ce qui n'est pas le cas. 
Remarque. Il est important de remarquer que le lemme 7.1 est "flexible": il peut en fait être étendu aux courbes $\gamma$ qui sont " $\epsilon$-elliptiques" au sens ou d'une part elles vérifient, pour un $\epsilon>0$ :

$$
\forall t \in[0, L],\left\langle\gamma^{\prime}(t) \mid \gamma^{\prime}(t)\right\rangle=1 \text { et }\left\langle\gamma^{\prime \prime}(t) \mid \gamma^{\prime \prime}(t)\right\rangle \leq \epsilon
$$

et ou d'autre part leur accélération $\gamma^{\prime \prime}$ reste hors de l'une des composantes connexes de l'ensemble des vecteurs de type temps de $T S_{1}^{n}$; leur longueur est alors au moins $2 \pi-A(\epsilon)$, ou $A$ est une fonction continue valant 0 en 0 . On peut démontrer ce résultat à peu près comme le lemme 7.1, mais, à la dernière étape, il faut choisir le point à partir duquel on cherche le $S^{n}$ de référence avec plus de soin, et on ne peut éviter le cas où $\gamma$ est incluse dans une hypersurface totalement géodésique (on utilise alors une minoration de la longueur des courbes à courbure majorée par $\epsilon$ dans $\left(S^{n}\right.$, can $)$ ).

On déduit du corollaire 7.2 une sorte de réciproque à la proposition 5.8:

Théorème 7.3. Soit $\sigma$ une métrique sur $S^{2}$; alors $\left(S^{2}, \sigma\right)$ admet une immersion isométrique $f$ uniformément elliptique dans $S_{1}^{3}$ si et seulement si

- $\left(S^{2}, \sigma\right)$ n'admet pas de géodésique fermée de longueur $L \leq 2 \pi$;

- la courbure de $\left(S^{2}, \sigma\right)$ vérifie partout $K<1$.

Il existe alors exactement deux telles immersions distinctes admettant un 1-jet donné en un point donné, et ce sont des plongements qui se déduisent l'un de l'autre par une isométrie globale de l'espace.

On peut voir ceci comme une sorte de version régulière du théorème d'Andreev (cf [Vin93]) ; en effet, immerger dans $H^{3}$ un polyèdre dont les angles entre les faces sont données revient à immerger dans $S_{1}^{3}$ un polyèdre dont la "métrique" est donnée (la dualité entre $H^{3}$ et $S_{1}^{3}$ dont il est question dans l'introduction s'étend à ce cas, cf [RH93]) et le théorème 7.3 donne un résultat dans le cas où le polyèdre est remplacé par une surface lisse.

Ce résultat a d'ailleurs été proposé par C. D. Hodgson et I. Rivin dans la conclusion de [RH93], qui contient un théorème similaire dans le cas où la surface $S$ est polyèdrale ; mais les démonstrations de [RH93], qu'on pourra aussi trouver dans [Riv86], reposent sur la dualité entre $S_{1}^{3}$ et $H^{3}$ et sont très différentes de celles qu'on donne ici.

Le corollaire 7.2 admet en réalité une généralisation à un cas beaucoup plus large; en fait, son analogue reste vrai pour une immersion riemannienne (c'est à dire de type espace) elliptique $\phi$ d'une variété de dimension $\mathrm{k}$, soit 
$V^{k}$, dans $S_{1}^{n}(k<n)$. On doit dans ce cas comprendre "elliptique" au sens de:

$$
\forall v \in T V^{k},\left\langle I\left(\phi_{*}(v), \phi_{*}(v)\right) \mid \Pi\left(\phi_{*}(v), \phi_{*}(v)\right)\right\rangle<0
$$

II désignant la deuxième forme fondamentale qui est maintenant une 2forme sur $T V$ à valeurs dans $N V$, le fibré normal sur $V$ induit par $\phi$. Plus précisément, on a dans ce cas le résultat suivant (dont la lecture n'est pas nécessaire à la compréhension de ce qui suivra):

Théorème 7.4. Soit $\phi: V^{k} \rightarrow S_{1}^{n}$ une immersion elliptique d'une variété compacte dans $S_{1}^{n}$ (avec $n \geq k$ ). La courbure sectionnelle de $\left(V, \phi^{*} g_{0}\right)$ est partout inférieure strictement à 1 , et on a: $\operatorname{Vol}_{k}(V) \geq \operatorname{Vol}_{k}\left(S^{k}\right.$, can $)$.

Démonstration. Nous allons procéder en plusieurs étapes:

- La courbure sectionnelle $K_{V}$ de $V$ est strictement majorée par 1. Soit en effet $v \in V$, et $P \subset T_{v} V$ un 2-plan. Notons $\left(e_{i}\right)_{i \in\{0, \ldots, n-k\}}$ une base orthogonale de $N_{v} V$, telle que $\left\langle e_{i} \mid e_{i}\right\rangle=\epsilon_{i}$ avec $\epsilon_{0}=-1$ et $\epsilon_{i}=1, i \geq \pi$. Notons $\Pi_{i}$ les composantes de $\Pi_{\mid P}$ dans cette base; les $\Pi_{i}$ sont donc des formes quadratiques sur $P$, et pour $x, y \in T_{v} V, I(x, y)=\sum_{i} I(x, y) e_{i}$. D'après la formule de Gauss, si $x, y$ sont linéairement indépendants dans $P$, alors

$$
K(P)=1+\frac{\sum_{i=0}^{n-k} \epsilon_{i}\left(\Pi_{i}(x, x) \Pi_{i}(y, y)-\Pi_{i}(x, y)^{2}\right)}{\langle x \mid x\rangle\langle y \mid y\rangle-\langle x \mid y\rangle^{2}}
$$

donc

$$
K(P)=1+\sum_{i=0}^{n-k} \epsilon_{i} \operatorname{det} \Pi_{i}
$$

Mais par ellipticité de $V$, on sait que

$$
\forall x \in P, \Pi_{0}(x, x)^{2}>\sum_{i=1}^{n-k} \Pi_{i}(x, x)^{2}
$$

Choisissons donc une base orthonormée $(x, y)$ de $P$ qui diagonalise $\Pi_{0}$. On a:

$$
\operatorname{det} \Pi_{0}=\Pi_{0}(x, x) \Pi_{0}(y, y)
$$

donc

$$
\operatorname{det} \Pi_{0}>\left[\sum_{i=1}^{n-k} \Pi_{i}(x, x)^{2}\right]^{\frac{1}{2}}\left[\sum_{i=1}^{n-k} \Pi_{i}(y, y)^{2}\right]^{\frac{1}{2}}
$$


grâce à 7.5 , donc

$$
\operatorname{det} \Pi_{0}>\sum_{i=1}^{n-k} \Pi_{i}(x, x) \Pi_{i}(y, y)
$$

par Cauchy-Schwarz, et donc finalement

$$
\operatorname{det} \Pi_{0}>\sum_{i=1}^{n-k} \operatorname{det} \Pi_{i}
$$

car $(x, y)$ est une base orthonormée de $P$. Donc $K(P)<1$ par (7.3).

- Le rayon d'injectivité $i$ de $V$ est minoré par $\pi$. On suit un raisonnement classique qu'on peut trouver dans [CE75], lemme 6.6. Soit en effet $p, q \in V$ tels que $q$ est dans le "cut locus" de $p$ et $\mathrm{d}(p, q)=i$. Alors de deux choses l'une: soit $p$ et $q$ sont conjugués le long d'une géodésique minimisante $\gamma$, soit il existe deux géodésiques $\gamma, \tilde{\gamma}$ distinctes avec $\gamma(0)=p=\gamma^{\prime}(0), \gamma(i)=q=\gamma^{\prime}(i)$. Mais la première possibilité est exclue, car (cf. [CE75], 1.29 par exemple) si $K_{V}<1$ alors exp est non-singulière sur les boules de rayon $r<\pi$ des $T_{v} V$. Dans le second cas, si $\gamma^{\prime}(0)+\tilde{\gamma}^{\prime}(0) \neq 0$ alors on peut trouver un segment géodésique $\sigma$ avec $\sigma(0)=p$, et $\left\langle\sigma^{\prime}(0) \mid \gamma^{\prime}(0)\right\rangle>0,\left\langle\sigma^{\prime}(0) \mid \tilde{\gamma}^{\prime}(0)\right\rangle>0$; on peut alors construire des familles $\gamma_{s}$ et $\tilde{\gamma}_{s}$ à un paramètre de géodésiques allant de $\sigma(s)$ à $q$, et pour $s$ assez petit on doit avoir (quitte à échanger $\gamma$ et $\tilde{\gamma})$ :

$$
L\left(\gamma_{s}\right) \leq L\left(\tilde{\gamma}_{s}\right)<L(\gamma)=L(\tilde{\gamma})
$$

ce qui impliquerait que $L\left(\tilde{\gamma}_{s}\right) \geq \pi$, ce qui est impossible d'après le corollaire 7.2. Donc $\gamma^{\prime}(0)+\tilde{\gamma}^{\prime}(0)=0$, et de même en $q$, ce qui implique que $\gamma$ et $\tilde{\gamma}$ forment ensemble une géodésique fermée de longueur inférieure à $2 \pi$, ce qui est impossible. Donc $i \geq \pi$.

- Le volume de $V$ est au moins $\operatorname{Vol}_{k}\left(S^{k}\right.$, can). Il suffit de comparer le volume d'une boule géodésique de rayon $\pi$ dans $V$ avec celui de $\left(S^{k}\right.$, can). En effet, si $v \in V$, et si $B_{r}^{T}(v)$ désigne la boule de centre $v$ et de rayon $r$ dans $T_{v} V$, on a:

$$
\operatorname{Vol}_{k}(V) \geq \operatorname{Vol}_{k}\left(B_{\pi}(v)\right)=\int_{B_{\pi}^{T}(v)} \operatorname{Jac}\left(T_{x} \exp _{v}\right) d x
$$

et donc

$$
\operatorname{Vol}_{k}(V) \geq \int_{S^{k-1}} \int_{0}^{\pi} \sin (r)^{k-1} d r d \sigma=\operatorname{Vol}_{k}\left(S^{k}, \text { can }\right)
$$


Là encore, le raisonnement est usuel (voir par exemple [BC64]).

Dans une direction un peu différente, on peut donner une autre conséquence du lemme 7.1, concernant plus précisément la possibilité d'immerger isométriquement une variété dans $S_{1}^{n+1}$ sous des hypothèses géométriques. On a ainsi le

Théorème 7.5. Soit $\left(V^{n}, \nu\right)$ une variété riemannienne (connexe) de dimension $n$ (éventuellement à bord, ouverte,etc...) à courbure de Ricci vérifiant :

$$
\forall v \in V, \forall x \in T_{v} V, \operatorname{Ric}(x, x)<(n-1) \nu(x, x)
$$

Supposons que $(V, \nu)$ admet une géodésique fermée de longueur $L \leq 2 \pi$. Alors $(V, \nu)$ n'admet pas d'immersion isométrique dans $S_{1}^{n+1}$.

Démonstration. Commençons par remarquer que, si $\phi: V \rightarrow S_{1}^{n+1}$ est une immersion isométrique, alors son image est nécessairement convexe. La formule de Gauss nous montre en effet que le tenseur de courbure $R$ de $\nu$ vérifie, pour tout $v \in V$ et pour tout $x, y \in T_{v} V$ orthogonaux et unitaires :

$$
\nu(R(x, y) x, y)=1-\Pi(x, x) \Pi(y, y)+\Pi(x, y)^{2}
$$

Si on note $\left(e_{i}\right)_{i \in\{1, . ., n\}}$ une base orthonormée de $T_{v} V$ qui diagonalise $I I$ et $\left(\lambda_{i}\right)_{i \in\{1, ., n\}}$ les valeurs propres associées, on voit que la courbure de Ricci de $V$ vérifie pour chaque $i \in N_{n}$ :

$$
\operatorname{Ric}\left(e_{i}, e_{i}\right)=(n-1)-\Pi\left(e_{i}, e_{i}\right) \sum_{j} \Pi\left(e_{j}, e_{j}\right)+\Pi\left(e_{i}, e_{i}\right)^{2}
$$

et donc en notant $h=\sum_{j} I\left(e_{j}, e_{j}\right)$ la trace de $I$ on obtient que pour $i \in N_{n}$ :

$$
\lambda_{i}\left(h-\lambda_{i}\right)>0
$$

ce qui montre que si $\lambda_{i}>0, h-\lambda_{i}>0$ et donc $h>0$ et que si $\lambda_{i}<0$ alors de même $h<0$ aussi, et donc que les $\lambda_{i}$ sont tous de même signe. De plus, ce signe est constant sur $V$ par continuité, et $\phi(V)$ est convexe.

On peut maintenant appliquer directement le lemme 7.1 et en déduire que les géodésiques fermées de $(V, \nu)$ sont nécessairement de longueur strictement plus grande que $2 \pi$. 
Surfaces convexes dans des espaces lorentziens à courbure constante 323

\section{Immersions de surfaces complètes.}

Nous allons voir comment utiliser les résultats précédents pour prouver des résultats d'existence (mais sans unicité) d'immersions isométriques dans $S_{1}^{3}$ de domaines de $S^{2}$ munis de métriques complètes vérifiant des conditions naturelles. Voici d'abord un lemme technique sur les recollements de surfaces:

Lemme 8.1. Soit $\epsilon>0,\left(S_{1}, \sigma_{1}\right)$ et $\left(S_{2}, \sigma_{2}\right)$ des surfaces dont les courbure vérifient $K_{1}, K_{2} \leq K_{0}, C_{1}, C_{2}$ des composantes connexes régulières de $\partial S_{1}$ et de $\partial S_{2}$, et $f: C_{1} \rightarrow C_{2}$ une isométrie. Notons $k_{1}, k_{2}$ les courbures de $C_{1}, C_{2}$ (positives aux points où les bords des surfaces sont convexes). Supposons que

$$
\forall c \in C_{1}, k_{1}(c)+k_{2}(f(c)) \leq 0
$$

Alors il existe une surface $(S, \sigma)$ à courbure $K \leq K_{0}+\epsilon$ et des difféomorphismes $\phi_{i}: S_{i} \rightarrow S(i=1,2)$ isométriques sur les $S_{i} \backslash\left(C_{i}\right)_{\epsilon}$, tels que

$$
\begin{gathered}
\phi_{1}\left(S_{1}\right) \cup \phi_{2}\left(S_{2}\right)=S \\
\phi_{1}\left(S_{1}\right) \cap \phi_{2}\left(S_{2}\right)=C=\phi_{1}\left(C_{1}\right)=\phi_{2}\left(C_{2}\right) \\
\left(\phi_{2 \mid C_{2}}\right)^{-1} \circ \phi_{1 \mid C_{1}}=f
\end{gathered}
$$

Si de plus $L(C)>L_{0},\left(S_{1}, \sigma_{1}\right)$ et $\left(S_{2}, \sigma_{1}\right)$ n'ont pas de géodésique (fermée) de longueur $L \leq L_{0}$, et s'il n'existe pas de segment géodésique de $\left(S_{2}, \sigma_{2}\right)$ entre 2 points de $\partial\left(\left(C_{2}\right)_{\epsilon}\right) \backslash C_{2}$ de longueur $L \leq L_{0}$, alors on peut choisir $(S, \sigma)$ sans géodésique (fermée) de longueur $L \leq L_{0}-\epsilon$.

Démonstration. Pour le premier point, il suffit de définir $S$ en collant les variétés $S_{1}, S_{2}$ le long de $C_{1}, C_{2}$ de manière naturelle. On obtient alors une métrique $\bar{\sigma}$ continue sur $S \operatorname{par} \sigma_{1}, \sigma_{2}$. On remarque que comme $k_{1}+k_{2} \leq 0$, $\bar{\sigma}$ a une courbure singulière négative ou nulle le long de $C$, et donc par régularisation de $\bar{\sigma}$ dans un $\epsilon$-voisinage de $C$ (cf. [AZ67] pour les problèmes de régularisation de surfaces singulières, etc) on obtient la métrique régulière $\sigma$ recherchée. Pour le second point, pour tout $\epsilon^{\prime}>0$, on peut faire la régularisation de $\bar{\sigma}$ de manière que toute géodésique de $\left(S_{1}, \sigma_{1}\right)$ rencontrant $\partial\left(\left(C_{1}\right)_{\epsilon}\right) \backslash C_{1}$ avec un angle plus grand que $\epsilon^{\prime}$ se prolonge en une géodésique de $(S, \sigma)$ rencontrant $\partial\left(\left(C_{2}\right)_{\epsilon}\right) \backslash C_{2}$. Mais si $\gamma$ est une géodésique fermée de $(S, \sigma)$, trois cas seront alors possibles:

- Soit $\gamma \subset S_{1}$ ou $\gamma \subset S_{2}$, et alors $L(\gamma) \geq L_{0}-\epsilon$ par hypothèse; 
- Soit $\gamma$ rencontre $\partial\left(C_{1}\right)_{\epsilon}$ avec un angle plus grand que $\epsilon^{\prime}$, mais alors $\gamma$ se prolonge en une géodésique de $(S, \sigma)$ rencontrant $\partial\left(C_{2}\right)_{\epsilon}$ et donc $\gamma$ contient un segment géodésique entre 2 points de $\partial\left(C_{2}\right)_{\epsilon} \backslash C_{2}$ et $\gamma$ est encore de longueur $L \geq L_{0}-\epsilon$;

- Soit $\gamma$ rencontre $\partial\left(\left(C_{1}\right)_{\epsilon}\right) \backslash C_{1}$ avec un angle plus petit que $\epsilon^{\prime}$, soit $\gamma$ est contenue dans $C_{\epsilon}$, et dans les 2 cas (pour $\epsilon, \epsilon^{\prime}$ assez petits) $L(\gamma) \geq$ $L(C)-\epsilon \geq L_{0}-\epsilon$.

Voyons ensuite le cas où la surface est compacte à bord, et ramenons nous au cas compact (sans bord) grâce au lemme 8.1:

Lemme 8.2. Soit $(\Omega, \omega)$ une surface riemannienne compacte à bord régulier telle que:

1. $\Omega$ admet un plongement régulier $\phi$ dans $S^{2}$;

2. $K(\omega)<1-\epsilon$;

3. $(\Omega, \omega)$ n'admet pas de géodésique fermée de longueur $L \leq 2 \pi+\epsilon$;

4. le bord $\partial \Omega=\bigcup_{i=1}^{p} C_{i}$ de $\Omega$ est tel que, si on note $k_{i}$ la courbure de $C_{i}$, qui est positive quand le bord est convexe, on ait pour tout $i \in$ $\{0, \ldots, p\}$ soit $L\left(C_{i}\right)>2 \pi$ et $k_{i} \leq 0$, soit $k_{i}>0$

Alors on peut compléter $\omega$ en une métrique $\sigma$ sur $S^{2}$ telle que $\phi^{*} \sigma=\omega$, et qui vérifie (2) et (3) ci-dessus.

Démonstration. Il suffit de faire la prolongation séparément sur chaque composante connexe de $\partial \Omega$. Choisissons donc une telle composante connexe et notons la $C$. Nous allons faire la démonstration en séparant les deux cas qui se trouvent dans l'hypothèse (4):

1. $L(C)>2 \pi$ et $k \leq 0$ : on peut trouver une application isométrique $\phi$ de $C$ dans une courbe régulière $\tilde{C}$ de $\left(S^{2}, \epsilon \operatorname{can}_{S^{2}}\right)$ ( $\epsilon$ petit) dont la courbure géodésique vérifie $\tilde{k}(\phi(s))+k(s)<0, k$ étant négative quand la convexité de $\tilde{C}$ est vers la composante connexe $\tilde{C}_{1}$ de $S^{2} \backslash \tilde{C}$ de plus grande aire. On peut alors "coller" $\Omega$ et $\tilde{C}_{1}$ le long de $C$ et de $\tilde{C}$, et on obtient une surface $\tilde{S}$ singulière le long d'une courbe $C$. Mais comme $\tilde{k}+k<0$, la courbure singulière de $\tilde{S}$ est négative et d'après le lemme 
8.1, on peut régulariser $\tilde{S}$ et obtenir une surface $S_{1}$ à courbure $K<1$ partout (voir [AZ67] pour ces problèmes). De plus, on voit que si $\epsilon$ est petit, $S_{1}$ n'a pas de géodésique fermée de longueur $L \leq 2 \pi$ puisque $\mathrm{L}(C)>2 \pi$.

2. $k>0$ : Prolongeons $\phi_{*} \omega$ en une métrique $C^{\infty}$ en choisissant une métrique $\tilde{\sigma}$ sur la composante $S_{C}$ de $S^{2} \backslash C$ disjointe de $\Omega$ (il est clair que c'est possible, par exemple par un prolongement non régulier suivi d'une régularisation par convolution sur $S^{2}$ ). Notons $\tilde{K}$ et $\tilde{L}$ la borne supérieure de la courbure et la borne inférieure de la longueur des géodésiques fermées sur la variété obtenue; choisissons $\epsilon^{\prime}>0$.

Notons encore $k$ la courbure géodésique de $C$, et choisissons $x \in C$. Posons

$$
\theta=\frac{1}{L(C)} \int_{C} k(\tau) d \tau
$$

On peut alors définir une fonction

$$
\begin{gathered}
\lambda: C \rightarrow R \\
y \mapsto \int_{x}^{y} k(\tau) d \tau-\theta d_{C}(x, y)
\end{gathered}
$$

$d_{C}$ désignant la distance orientée sur $C$, puis définir une métrique $\bar{\sigma}$ sur $C \times\left[0, \frac{1}{\epsilon^{\prime}}\right]$ par

$$
\bar{\sigma}=d r^{2}+(1+\theta r) \cos (\lambda(y)) d r . d y+(1+\theta r)^{2} d y^{2}
$$

$y$ désignant la coordonnée sur $C$.

On vérifie que $\bar{\sigma}$ est une métrique plate, et que la courbure géodésique de $C \times\{0\}$ est $-k$, ce qui montre qu'on peut définir une variété sans courbure singulière en collant $(S, \sigma)$ et $\left(C \times\left[0, \frac{1}{\epsilon^{\prime}}\right], \bar{\sigma}\right)$ le long de $C$ et de $C \times\{0\}$ puis $\left(C \times\left[0, \frac{1}{\epsilon^{\prime}}\right], \bar{\sigma}\right)$ et $\left(S_{C},\left(\frac{\epsilon^{\prime}}{1+\epsilon^{\prime}}\right)^{2} \tilde{\sigma}\right)$ le long de $C \times\left\{\frac{1}{\epsilon^{\prime}}\right\}$ et de $C$. De plus, si $\epsilon^{\prime}<\frac{1}{\tilde{K}}$, la métrique obtenue a une courbure $K<1-\epsilon$, et on peut la régulariser en maintenant cette condition. Enfin, toujours si on choisit $\epsilon^{\prime}$ assez petit (cette fois avec en particulier $\epsilon^{\prime}<\frac{1}{\tilde{L}}$ ) elle n'aura pas de géodésique de longueur $L \leq 2 \pi-\epsilon$ car une telle géodésique devrait soit être dans $S$ (car $k>0$, donc $S$ est convexe et une géodésique sortant de $S$ doit passer par $S_{C}$; mais alors sa longueur vérifie $L>2 \pi$ pour $\epsilon^{\prime}$ petit), soit être dans un voisinage de $S_{C}$ (ce qui est impossible pour $\epsilon^{\prime}$ petit). 
Corollaire 8.3. Dans les conditions du lemme 8.2, $(\Omega, \omega)$ admet au moins deux immersions isométriques dans $S_{1}^{3}$ ayant un 1-jet donné en un point donné.

Démonstration. Il suffit d'utiliser le lemme 8.2 pour prolonger la métrique en une métrique convenable sur $S^{2}$, puis le théorème 6.6 pour obtenir deux immersions isométriques de $S^{2}$ dans $S_{1}^{3}$, chacune correspondant à un coté du plan tangent au point où le 1-jet est fixé (cf. la remarque suivant le lemme 6.6 .

Nous allons maintenant appliquer le corollaire 8.3 pour trouver un résultat d'existence d'immersion pour des surfaces complètes (non compactes). On va pour cela compléter la notion de longueur de la plus courte géodésique fermée en tenant compte de ce qui peut se passer à l'infini:

Définition 8.4. Soit $(S, \sigma)$ une surface complète munie d'une métrique riemannienne et $s \in S$. Pour $x \in S$, on note $L_{x}$ la longueur de la plus courte boucle non homotope à $\{x\}$ passant par $x$. On peut alors poser

$$
E((S, \sigma))=\inf _{x \in S} L_{x}
$$

$E((S, \sigma))$ est une sorte d'équivalent à l'infini de la longueur de la plus courte des géodésiques fermées.

Nous pouvons maintenant trouver une classe de surfaces complètes admettant une immersion isométrique elliptique dans $S_{1}^{3}$ :

Théorème 8.5. Soit $(\Omega, \omega)$ une surface riemannienne complète telle que:

1. $\Omega$ admet un plongement régulier dans $S^{2}$;

2. $K(\omega) \leq 1-\epsilon$ pour un certain $\epsilon>0$;

3. $(\Omega, \omega)$ n'admet pas de géodésique fermée de longueur $L \leq 2 \pi+\epsilon$;

4. $E((S, \sigma))>2 \pi$.

Alors $(\Omega, \omega)$ admet au moins deux immersions isométriques elliptiques dans $S_{1}^{3}$ ayant un 1-jet donné en un point donné.

Démonstration. On va procéder en plusieurs étapes pour utiliser les résultats précédents. 
- Choisissons $x_{0} \in \Omega$. Si $x \in S$ et $d\left(x, x_{0}\right)$ est assez grand, il existe une courbe fermée non homotope à $\{x\}$, passant par $x$, et dont la courbure $k$ vérifie partout soit $k \leq 0$, soit $k>0$ ( $k$ étant pris négatif quand la convexité de la courbe est vers $\left.x_{0}\right)$. On peut en effet trouver une géodésique $\gamma:[0, L] \rightarrow S$ avec $\gamma(0)=\gamma(L)=x$ (il suffit de prendre la plus courte des courbes passant par $x$ dans une classe d'homotopie donnée; puis il suffit de la régulariser en "étalant" l'angle qu'elle fait en $x$ sur le reste de la boucle, ce qui est possible en déplaçant chacun de ses point de moins de $\epsilon$ pour tout $\epsilon>0$ donné.

- Il existe une suite croissante $\left(K_{n}\right)_{n \in N}$ de domaines compacts (à bord) de $\Omega$, vérifiant les conditions du lemme 8.2, tels que $\cup_{n \in N} K_{n}=\Omega$. En effet, le seul problème est de choisir les $K_{n}$ tels que leurs bords vérifient la condition $4 \mathrm{du}$ lemme 8.2 , mais c'est possible sans quoi $\Omega$ aurait des "branches" qu'on ne peut pas "couper" par des courbes $C$ ayant soit $L(C)>2 \pi$ et $k \leq 0$, soit $k>0$. Or pour tout $x \in S$, il existe une courbe $\gamma$ simple non homotope à $\{x\}$ passant par $x$ et à courbure $k$ de signe constant; comme $E((\Omega, \omega))>2 \pi$, cette courbe a une longueur $L>2 \pi$, et suivant que $k>0$ ou $k \leq 0$, on est dans l'une des deux versions de l'hypothèse (4) du lemme 8.2 , et on peut utiliser $\gamma$ pour "couper" $\Omega$ en deux composantes connexes (car $\Omega$ admet un plongement dans $S^{2}$ ).

- Si $x_{0} \in K_{0}$ et $I \in \operatorname{Isom}\left(T_{x_{0}} \Omega, S_{1}^{3}\right)$ alors les $\left(K_{n}, \omega_{\mid K_{n}}\right)$ admettent chacun deux immersions isométriques $f_{n}^{1}$ et $f_{n}^{2}$ dans $S_{1}^{3}$ telles que $j^{1} f_{n}^{i}\left(x_{0}\right)=I$ pour $i \in\{1,2\}$. Il suffit en effet d'appliquer le lemme 8.2 .

- Il existe des sous-suite des $\left(\tilde{f}_{n}^{i}\right)=\left(j^{1} f_{n}^{i}\right)$ qui convergent (comme suites d'applications de $K_{n}$ dans $E$ ) vers des immersions pseudoholomorphes $\tilde{f}_{\infty}^{i}$; de plus, $\tilde{f}_{\infty}^{1} \neq \tilde{f}_{\infty}^{2}$ car leur comportement est différent au voisinage du point de $K_{0}$ où le 1 -jet est fixé (les $\tilde{f}_{n}^{1}$ correspondent à des immersions se trouvant d'un coté du plan tangent en ce point, les $\tilde{f}_{n}^{2}$ de l'autre). Il faut pour le voir raisonner comme dans la démonstration du théorème 5.5. Ces limites sont alors des 1-jet d'immersions isométriques, car les plis sont exclus par la géométrie $\operatorname{des}\left(K_{n}, \omega_{\mid K_{n}}\right)$.

- D'après la condition (3) (et le fait que $(\Omega, \omega)$ est complète), les $\tilde{f}_{\infty}^{i}$ ne peuvent être inclus dans une surface de pli, et correspondent donc à des immersions isométriques de $(\Omega, \omega)$ dans $S_{1}^{3}$. Cf. le théorème 5.6. 
Le fait d'imposer que $\Omega$ admette un plongement régulier dans $S^{2}$ est nécessaire pour que notre construction fonctionne: en effet, sinon, il existe un domaine compact de $\Omega$ qui n'admet pas de tel plongement dans $S^{2}$, et nos découpages et recollements ne nous conduiront pas à des sphères (seules surfaces compactes de type espace immergées). A l'opposé, il existe des surfaces de type espace complètes immergées dans $S_{1}^{3}$ dont la projection "orthogonale" sur un $S^{2}$ totalement géodésique n'est pas un difféomorphisme global; de telles surfaces ne peuvent pour la même raison pas être obtenues par notre méthode.

Réciproquement, le théorème suivant montre que la condition imposée $\operatorname{sur} E((S, \sigma))$ n'est pas déplacée:

Théorème 8.6. Soit $(S, \sigma)$ une surface complète telle que $E((S, \sigma))<2 \pi$. Alors $(S, \sigma)$ n'admet pas d'immersion isométrique elliptique dans $S_{1}^{3}$.

Démonstration. Supposons que $E((S, \sigma))=2 \pi-2 \epsilon$. Si $L_{x}$ admet un minimum local $m<2 \pi$ en un point $x_{0} \in S$, alors il existe une géodésique passant par $x_{0}$ de longueur $m$, et le lemme 7.1 nous montre que $(S, \sigma)$ n'admet pas d'immersion isométrique dans $S_{1}^{3}$.

Sinon, on peut trouver une géodésique $\gamma: R_{+} \rightarrow S$ (parametrée par la longueur d'arc) partant à l'infini telle que $L: t \mapsto L_{\gamma(t)}$ soit une fonction décroissant vers $2 \pi-2 \epsilon$.

Soit $x_{0}$ le point de $\gamma$ tel que $L_{x_{0}}=2 \pi-\epsilon$, et $B$ la fonction de Buseman :

$$
\begin{aligned}
B: S & \rightarrow R \\
& x \mapsto \lim _{t \rightarrow \infty} d_{\sigma}\left(\gamma(t), x_{0}\right)-d_{\sigma}(\gamma(t), x)
\end{aligned}
$$

Choisissons $\epsilon^{\prime}>0$, prenons une fonction $C^{\infty}$ croissante $\psi: R \rightarrow R$ valant 1 pour $t \leq 0$ et $1+2 \epsilon$ pour $t \geq t_{0}>0$ en ayant partout une dérivée majorée par $\epsilon^{\prime} / 4$. Notons enfin $\tilde{\sigma}=(\psi \circ B)^{2} \sigma$.

Alors par construction il existe pour $\tilde{\sigma}$ une géodésique fermée de longueur $L \leq \pi-\epsilon$ correspondant à un minimum local de la fonction $\tilde{L}_{x}$ définie comme la longueur pour $\tilde{\sigma}$ de la plus courte courbe non homotope à 0 passant par $x$. Et comme le gradient de $\psi \circ B$ est majoré par $\epsilon^{\prime} / 4$, cette géodésique pour $\tilde{\sigma}$ a aussi une courbure (géodésique) majorée par $\epsilon^{\prime}$ pour $\sigma$ et sa longueur pour $\sigma$ est au plus $2 \pi-\epsilon$. 
On en déduit, en utilisant la remarque qui suit la démonstration du lemme 7.1 avec un $\epsilon^{\prime}$ assez petit, que $(S, \sigma)$ n'admet pas d'immersion isométrique dans $S_{1}^{3}$.

Contrairement aux résultats d'existence d'immersions isométriques, ce résultat peut se démontrer en utilisant un résultat parallèle de [RH93] ; réciproquement, on peut démontrer le résultat polyèdral en utilisant ce résultat lisse. Par contre, les démonstrations sont très différentes.

Il est d'ailleurs facile de voir que le théorème 7.5 permet de montrer un résultat analogue sous des hypothèses de courbure de Ricci :

Théorème 8.7. Soit $\left(V^{n}, \nu\right)$ une variété de dimension $n$ à courbure de Ricci inférieure strictement à $(n-1) \nu$ et telle que $E((V, \nu))<2 \pi$. Alors $\left(V^{n}, \nu\right)$ n'admet pas d'immersion isométrique dans $S_{1}^{n+1}$.

La démonstration est la même que pour le théorème précédent : on fait apparaître une courbe de longueur $L<2 \pi$ qui est soit géodésique, soit à courbure géodésique suffisamment petite, et on peut alors montrer que son image par une immersion isométrique serait nécessairement une courbe elliptique de $S_{1}^{n+1}$, ce qui est interdit par le lemme 7.1.

Concluons en remarquant que les résultats présentés ici dans le cas d'immersions dans $S_{1}^{3}$ sont essentiellement valables pour tous les espace lorentziens de dimension 3 complets à courbure constante 1 . On sait en effet (voir [Wol74]) qu'il existe exactement 3 tels espaces : $S_{1}^{3}$, son quotient $R P_{1}^{3}$ par l'application antipodale, et un autre quotient, $Q_{1}^{3}$, par l'action de $Z_{2}$ qui, dans un modèle de $S_{1}^{3} \simeq S^{2} \times R$, envoie $(\sigma, u)$ sur $(-\sigma, u)$.

Or $Q_{1}^{3}$ peut s'écrire comme $R P^{2} \times R$ muni de la métrique $\operatorname{ch}(r)^{2} \operatorname{can}_{R P^{2}-}$ $d r^{2}$, donc les surfaces compactes qui y admettent des immersions de types espace sont homéomorphes soit à $S^{2}$ (elles ne sont alors pas plongées), soit à $R P^{2}$ (elles le sont alors nécessairement). Dans le premier cas, les immersions se relèvent en plongements dans $S_{1}^{3}$, et dans le second cas, ce sont leurs revêtements à deux feuillets qui admettent des plongements dans $S_{1}^{3}$. De plus, les immersions dans $Q_{1}^{3}$ sont elliptiques si et seulement si ces immersions déduites dans $S_{1}^{3}$ le sont, ce qui résoud le problème dans ce cas.

Par contre, $R P_{1}^{3}$ peut se voir comme l'espace total d'un fibré en droite "tordu" au dessus de $R P^{2}$; les surfaces qui y admettent des immersions de type espace sont encore homéomorphes soit à $S^{2}$, soit à $R P^{2}$. Dans le premier cas, elles ne sont pas plongées et les immersions se relêvent en plongements dans $S_{1}^{3}$ qui sont elliptiques si et seulement si elles le sont ; dans le second cas, par contre, elles ne peuvent pas être elliptiques, sans quoi on pourrait 
en déduire des plongements elliptiques de $S^{2}$ dans $S_{1}^{3}$ pour lesquelles deux points antipodaux sur $S^{2}$ seraient envoyés sur deux points antipodaux de $S_{1}^{3}$, avec des convexités dirigées vers deux cotés opposés de la surface; ce qui est impossible puisque l'immersion est elliptique.

On pourrait donc utiliser les résultats qui se trouvent plus haut pour caractériser toutes les immersions elliptiques de surfaces compactes dans des espaces lorentziens complets de dimension 3 à courbure constante 1 , et pour donner des résultats d'existences d'immersions (et de plongements) concernant les surfaces complètes.

\section{References.}

[And70] E.M. Andreev, Convex polyhedra in Lobacevskii space, Mat. Sb.(N.S.), 81 (123):445-478, 1970.

[AZ67] A. D. Aleksandrov and V. A. Zalgaller, Intrinsic Geometry of Surfaces, volume 15 of Translations of Mathematical Monographs, AMS, 1967.

[BC64] R.L. Bishop and R.J. Crittenden, Geometry of Manifolds, Academic Press, 1964.

[CD94] R. Charney and M. Davis, The polar dual of a convex polyhedral set in hyperbolic space, Preprint, 1994.

[CE75] J. Cheeger and D. G. Ebin, Comparaison Theorems in Riemannian Geometry, North Holland, 1975.

[CM62] E. Calabi and M. Markus, Relativistic space forms, Annals of Math. 75 (1962), 63-76.

[Gou96] E. Goursat, Leçon sur l'intégration des équations aux dérivées partielles du second ordre, volume 1, Hermann, 1896.

[Gro85] M. Gromov, Pseudo-holomorphic curves in symplectic manifolds, Invention. Math. 82 (1985), 307-347.

[Gro86] M. Gromov, Partial Differential Relations, Springer, 1986.

[Ham82] R. S. Hamilton, The inverse function theorem of Nash and Moser, Bull. Amer. Math. Soc. (N. S.), 7 (1982), 65-222.

[Lab] F. Labourie, Equations de Monge-Ampère elliptiques, en préparation.

[Lab87] F. Labourie, Limites d'hypersurfaces localement convexes, Invent. Math. 90 (1987), 115-138. 
Surfaces convexes dans des espaces lorentziens à courbure constante 331

[Lab89] F. Labourie, Immersions isométriques elliptiques et courbes pseudo-holomorphes, Journal of Differential Geometry, 30 (1989), 395-424.

[Lab94] F. Labourie, Exemples de courbes pseudo-holomorphes en géométrie riemannienne, In Audin and Lafontaine, editors, Pseudo-Holomorphic Curves in Symplectic Geometry, pages 251-270. Birkhauser, 1994.

[McD91] D. McDuff, The local structure of pseudo-holomorphic curves in almost complex 4-manifolds, Journal of Differential Geometry, 34 (1991), 143164.

[Nir53] L. Nirenberg, The Weyl and Minkowski problem in differential geometry in the large, Comm. Pure Appl. Math. 6 (1953), 337-394.

[O'N83] B. O'Neill, Semi-Riemannian Geometry, Academic Press, 1983.

[Pan] P. Pansu, Notes sur les pages 316 à 323 de l'article de m. gromov [6] pseudo-homorphic curves in symplectic manifolds, preprint.

[Pog73] A. V. Pogorelov, Extrinsic geometry of convex surfaces, technical report, Israel Program for Scientific Translation, 1973.

[RH93] I. Rivin and C. D. Hodgson, A characterization of compact convex polyhedra in hyperbolic 3-space, Invent. Math. 111 (1993), 77-111.

[Riv86] I. Rivin, Thesis, PhD thesis, Princeton University, 1986.

[SG85] D. D. Sokolov and V. G. Gaidalovich, Closed convex surfaces in pseudoEuclidean space, Dokl. Akad. Nauk USSR, 9 (1985), 9-10.

[Sok79] D. D. Sokolov, Closed surfaces having bounded total curvature in pseudoEuclidean space, Russian Math. Surveys, 34(3) (1979), 222-223.

[Vin93] E.B. Vinberg, editor, Geometry II, Geometry of Spaces of Constant Curvature, volume 29 of Encyclopaedia of Mathematical Sciences, Springer, 1993.

[Wol74] J. A. Wolf, Spaces of constant curvature, Publish or Perish, 1974.

RECEIVED MAY 31st, 1995.

Topologie et Dynamique

URA 169 DU CNRS

BATIMENT 425

UNIVERSITÉ DE PARIS-SUd

91405 ORSAY CEDEX

FRANCE 